\title{
再結晶現象における結晶核と母相に対する 混相理論としての保存則の定式化*
}

\author{
村松眞由 ${ }^{* 1}$ ，青柳吉輝 ${ }^{* 2}$ ，志澤一之*3
}

\author{
Formulation of Balance Laws as Mixture Theory \\ for Nuclei and Matrix in Recrystallization
}

\author{
Mayu MURAMATSU, Yoshiteru AOYAGI and Kazuyuki SHIZAWA*3 \\ ${ }^{* 3}$ Keio Univ. Dept. of Mechanical Engineering, \\ 3-14-1 Hiyoshi, Kohoku-ku, Yokohama, Kanagawa, 223-8522 Japan
}

In the previous work, the authors formulated the balance laws of mass, momentum, angular momentum and energy of the lattice element used for recrystallization. These laws were summed up over a phase in a representative volume element (RVE) and averaged in the RVE so as to develop the discrete balance laws for single phase. Furthermore, the balance law of angular momentum was separated into a bulk and a lattice parts through the orderestimation with the representative lengths both in macroscopic and microscopic scales. In this paper, the RVE converges on a material point so that the laws are rewritten in the integration form. When the laws are summed up all over the phases and averaged in them, the balance laws of mass, momentum, angular momentum and energy for nuclei and matrix as mixture are formulated, using an useful theorem proposed for the mixing summation of unsteady terms. At this time, the macroscopic part of the balance law for angular momentum results in the usual equation of angular momentum, so that the stress tensor keeps symmetry even if the lattice rotation is considered. While, the microscopic one is localized as an equation of spin angular momentum for lattice, which is suggested to be equivalent to the evolution equation of crystal orientation in KWC type phase-field model. Moreover, the increase law of entropy for mixture is also formulated. During this process, the entropy flux is defined by use of relative mass flux and chemical potential of phase transformation.

Key Words : Phase Transformation, Heat Treatment, Micromechanics, Recrystallization, Balance Law, Second Law of Thermodynamics, Mixture Theory, Phase-Field Model

\section{1. 緒 言}

圧延・焼鈍の最終工程で得られる金属材料の微視組織は材料の機械的性質や物性などの諸特性に多大な 影響を及ぼすため，焼鈍処理で生じる再結晶現象には大きな関心が寄せられている．再結晶過程における 各段階の現象については様々な報告(1) (3)がなされているものの，未だにそのメカニズムは十分に解明され ていないのが現状である。一方，近年では計算機の目覚ましい発展にともなって，数值解析による再結晶 予測に期待が寄せられており，Phase-fieldモデル(4) (7)を用いた再結晶シミュレーション(8) (12)が盛んに行われ ている. 従来のPhase-field理論では，エネルギー汎関数を用い，最小作用の原理に基づいて支配方程式を導 出しているが，一般に，エネルギ一原理には必ずそれと等価な保存則が存在することが知られている。こ れに対し，Phase-fieldモデルのエネルギー原理については未だにそのような保存則の存在は指摘されておら ず，特にKWC形Phase-fieldモデル(8)における結晶方位の支配方程式に等価な保存則がいかなるものである かも明らかになっていない. しかし，Phase-fieldモデルにおける支配方程式がどのような量の釣合い法則に 立脚するのかを知ることは学術的に重要である。また，様々な物質に対してその物性を変更してモデル化 する場合，構成式を介して熱力学的に議論するのが最も望ましいといえるが，エネルギ一原理には流束の

\footnotetext{
* 原稿受付 2012 年 1 月 21 日

*1 正員, 慶應義塾大学大学院理工学研究科（干223-8522 神奈川県横浜市港北区日吉 3-14-1）

*2 正員, (独) 日本原子力研究開発機構 原子力基礎工学研究部門 （干319-1195 茨城県那珂郡東海村白方白根 2-4）

*3 正員, 慶應義塾大学理工学部

E-mail: shizawa@mech.keio.ac.jp
} 
概念が存在しないため，構成式を介して物質特性を導入できないという難点を有している，一方，相変態 を伴う材料に対する熱力学的検討(13)(14) は以前から行われているが，いずれも場の中での連続的な方位発展 については考慮しておらず, 本研究で対象としている静的再結晶のような単純拡散変態を扱えるものはな い. 以上のような問題点を克服するためには，再結晶現象に対する保存則体系を定式化し，得られた保存 則に基づいてPhase-fieldモデルを再構築することは意義深い.

ところで，保存則を基礎とする従来の連続体の釣合い方程式は，内部構造を有する物質が一様・均質な 連続体であることを仮定して導出されており，原子・分子などの $\mathrm{nm}$ スケールや結晶粒構造のような $\mu \mathrm{m}$ ス ケールの挙動を考慮していない. 再結晶現象のように結晶格子スケールのミクロ挙動がマクロ場に影響を 及ぼすような現象を連続体力学的にモデル化する場合には, nmあるいは $\mu \mathrm{m}$ スケールの挙動を粗視化して 反映させる必要がある. そのような連続体力学の枠組みは一般化連続体力学 ${ }^{(15)}$ として体系化されており, その例として, ひずみこう配理論 ${ }^{(16)}$, n次物質理論 ${ }^{(17)}$, マイクロモルフィック理論 ${ }^{(18)(19)}$, マイクロポーラ

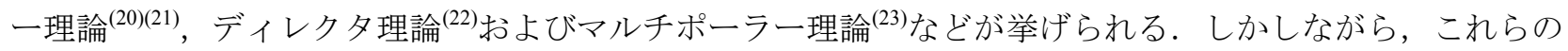
理論は共通して, 下部組織における内部回転などの変形の自由度が許容されるため, Cauchy応力の対称性 が損なわれるという問題点を有している．また，結晶格子スケールの物質湧き出しを考慮した混合理論(24) 形には拡張されていないため, 再結晶における結晶核(新相, 再結晶相または生成相) と母相の相互作用を 混合理論として取り扱えるモデルは存在しない.

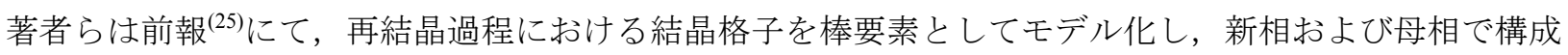
される材料において, 各相に属する結晶格子の微視的挙動の平均操作の結果として巨視的挙動を理論的に 関連づけ，単相に対する離散的保存則を定式化した。このとき，角運動量保存則およびエネルギー保存則 には結晶格子のスピン運動と代表体積要素 (RVE) 内での公転運動の情報が導入されたが, 対象となるバル クスケールおよび結晶格子スケールに特有の長さを代表量として選んでオーダー評価し, 角運動量保存則 をバルク部分および結晶格子部分に分離した。ただし, 前報で導出した保存則はRVE内における物理量に 平均化を施した離散的保存則であり, RVEは有限体積を有したままであるため, 連続体における保存則の 局所形は得られていない，また，それらは単相(再結晶相または母相)に対する保存則であり，混相(界面） に対する保存則を定式化するには至っていない.

そこで本報では，まず，前報で定式化した離散的保存則に対し，RVEを連続体の一物質点に帰着させる ことで, 単相に対する連続体としての質量, 運動量, 角運動量およびエネルギーの保存則を定式化する. また，得られた保存則を通常の手順で局所化する. 次に, 局所形の保存則を相にわたって和を取るととも に平均化操作を実施して混相に対する保存則を導く。このとき, 単相に対する各保存則の非定常項を複数 の相にわたって加算平均するための有用な定理を導出する.さらに, 得られた諸保存則に基づいて, エン トロピー増大則を定式化する。このとき, エントロピー流束は各相間の相対質量流束を用いて定義される. また，単相に対する質量保存則および混相に対する格子の角運動量保存則からそれぞれKWC形モデル(8)に おける秩序変数に対する釣合い方程式および結晶方位の釣合い方程式が得られることを示唆する．加えて， 混相に対するバルクの角運動量保存則からは通常の角運動量方程式が得られ, Cauchy応力は対称性を保つ ことを示す.

なお，相転移は一般的に物質の三態間の変化や鉄鋼材料における $\gamma$ 相から $\alpha$ 相への変化のような拡散変 態, あるいはマルテンサイト変態のような無拡散変態といった結晶構造の異なる相への変化として定義さ れる. 一方, 再結晶や粒成長のように相数および組成の変化を伴わない遷移現象は単純拡散変態といわれ,

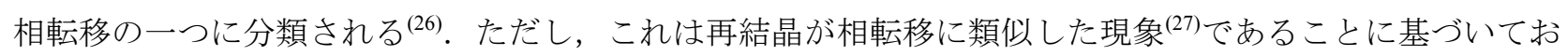
り, あくまでも広義の意味での相転移である. 本研究でもこの考えに従い, 再結晶を相転移として扱うこ とにする. また, 前報から始まった一連の研究は, KWC形Phase-fieldモデルの方位方程式の位置づけを明 確にすることを主な目的としているが，本報で定式化する保存則は一般原理であるため，結晶方位が連続 的に変化する単純拡散変態現象であればどのような材料にも適用可能である. したがって, そのような保 存則から得られる支配方程式もKWC形Phase-field方程式に限定されるものではなく, より汎用的なモデル への拡張も可能であることを付記しておく. 


\section{主 な 記 号}

本論文で用いる主な記号および特に混同しやすい記号を以下に列挙しておく.

$A \quad$ : 混相における任意の量の平均值

$A_{p} \quad:$ 相 $p$ における任意の量

$\hat{A}_{p} \quad$ : 平均值 $A$ からの $A_{p}$ の相対值

$\breve{A} \quad$ : 湧き出し量

$a_{a} \quad$ : 代表体積要素(六面体)の表面のうちの1つの面積

$\breve{b}_{p} \quad:$ 相 $p$ の体積質量涌き出し

$\boldsymbol{E} \quad$ : 微小ひずみテンソル

D ：変形速度テンソル

$\boldsymbol{F}_{p} \quad:$ 相 $p$ の変形こう配テンソル

$J_{p} \quad:$ 相 $p$ のヤコビアン

$\boldsymbol{J} \quad$ : 慣性モーメントテンソル

$\boldsymbol{j}_{p} \quad:$ 相 $p$ の相対質量流束ベクトル

$\boldsymbol{L} \quad$ ：混相の速度こう配テンソル

$\breve{l}_{p} \quad:$ 相 $p$ の相互作用角運動量ベクトル

$\boldsymbol{M} \quad$ : 混相の遇カテンソル

$\boldsymbol{M}_{\mathrm{I}} \quad$ : 各相の偶応力テンソルの単純和 $\left(\boldsymbol{M}_{\mathrm{I}} \equiv \sum_{p} \boldsymbol{M}_{p}\right)$

$\underset{(n)}{\boldsymbol{M}_{p}} \quad:$ 相 $p$ の遇応力テンソル $\left(\boldsymbol{m}_{p} \equiv \boldsymbol{M}_{p} \boldsymbol{n}\right)$

$\boldsymbol{m}_{p} \quad$ : 相 $p$ のスピンによる表面偶応力ベクトル

${\stackrel{(n)}{n_{p}}}_{n^{2}}$ : 相 $p$ の公転による表面偶応力ベクトル

$\breve{\boldsymbol{p}}_{p} \quad:$ 相 $p$ の相互作用運動量ベクトル

$q_{p} \quad:$ 相 $p$ の表面から供給される熱量

$q \quad$ : 混相の熱流束ベクトル

$\boldsymbol{q}_{\mathrm{I}} \quad$ : 各相の熱流束ベクトルの単純和 $\left(\boldsymbol{q}_{\mathrm{I}} \equiv \sum_{p} \boldsymbol{q}_{p}\right)$

$\boldsymbol{s}_{p} \quad:$ 相 $p$ のスピン角運動量ベクトル

$s \quad$ : 混相の角運動量密度ベクトル

$\boldsymbol{T}_{p} \quad:$ 相 $p$ の応力テンソル $\left(\boldsymbol{t}_{p} \equiv \boldsymbol{T}_{p} \boldsymbol{n}\right)$

$\boldsymbol{t}_{p} \quad:$ 相 $p$ の表面力(けん引力)べクトル

$\boldsymbol{T} \quad$ : 混相の応力テンソル

$\boldsymbol{T}_{\mathrm{I}}$ : 各相の応力テンソルの単純和 $\left(\boldsymbol{T}_{\mathrm{I}} \equiv \sum_{p} \boldsymbol{T}_{p}\right)$

$v_{a} \quad:$ 代表体積要素の体積

$\boldsymbol{v}_{p} \quad$ : 相 $p$ の物質速度ベクトル

$v \quad$ : 混相の物質速度ベクトル

$\boldsymbol{W}:$ 連続体スピンテンソル

$X_{p} \quad$ : 参照配置における相 $p$ の位置ベクトル

$\boldsymbol{x}$ : 物質点の位置ベクトル

$\breve{\varepsilon}_{p} \quad:$ 相 $p$ の相互作用エネルギー

$\varepsilon \quad$ : 混相の内部エネルギー密度

$\varepsilon_{\mathrm{I}}:$ 各相の内部エネルギー密度の単純和 $\left(\varepsilon_{\mathrm{I}} \equiv \sum_{p} \varepsilon_{p}\right)$

$\eta_{p} \quad:$ 相 $p$ のエントロピー密度 


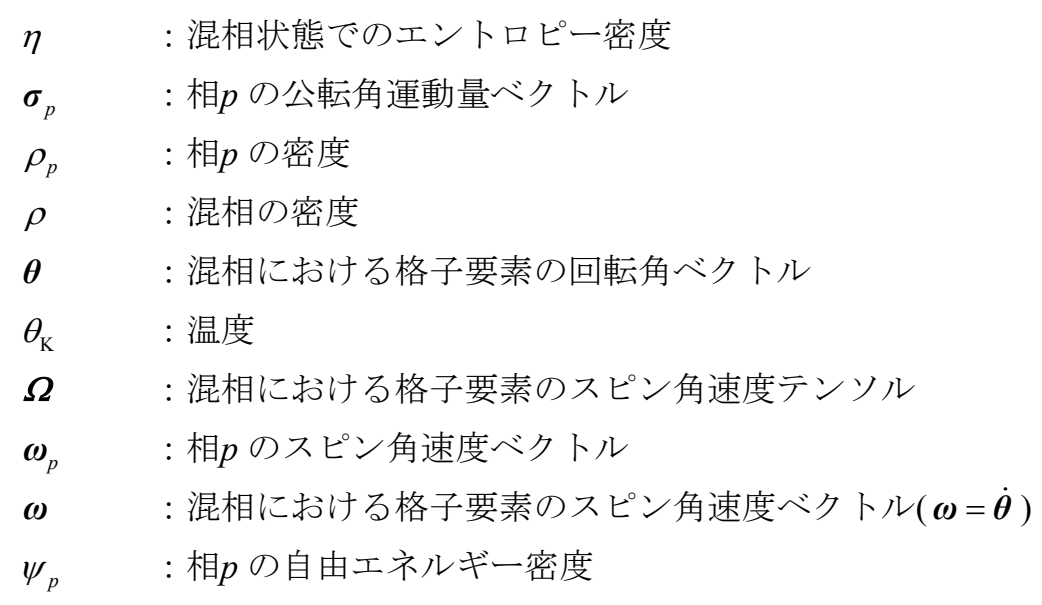

\section{2. 代表体積要素を基準とする保存則}

静的再結晶過程にある材料の再結晶相一母相界面においては，蓄積転位による格子の乱れと原子の熱振動 によって原子間力の釣合いが一部損なわれ，原子が安定位置に移ろうとして結晶格子は図1のように回転す る，そこで本研究では，図2のように両端にそれぞれ原子を1つずつ有する格子ユニットを，それと等価な 質量をもつ弾性棒としての格子要素でモデル化する(図3)，さらにこのような格子要素を多数含むRVEを検 查体積内に配置する (図4). 以上のモデルに基づき, 格子のスピン運動を考慮し, 相 $p$ におけるRVE内で平 均化すれば, 前報 ${ }^{(25)}$ で示したように質量, 運動量, バルクの角運動量, 格子の角運動量およびエネルギー の保存則がそれぞれ以下のように得られる.

$$
\begin{gathered}
\frac{d}{d t} \sum \rho_{p} v_{a}=\sum \breve{b}_{p} v_{a} \\
\frac{d}{d t} \sum \rho_{p} \boldsymbol{v}_{p} v_{a}=\sum\left(\sum_{n}^{(n)(n)} \boldsymbol{t}_{p} a_{a}+\breve{\boldsymbol{p}}_{p} v_{a}+\breve{b}_{p} \boldsymbol{v}_{p} v_{a}\right) \\
\frac{d}{d t} \sum \rho_{p}\left(\boldsymbol{x} \times \boldsymbol{v}_{p}+\boldsymbol{\sigma}_{p}\right) v_{a}=\sum\left[\sum_{n}\left(\boldsymbol{x} \times \stackrel{(n)}{p}+^{(n)} \boldsymbol{n}_{p}\right) a_{a}^{(n)}+\left(\boldsymbol{x} \times \breve{\boldsymbol{p}}_{p}+\breve{\boldsymbol{\mu}}_{p}+\breve{\boldsymbol{l}}_{p}\right) v_{a}+\breve{b}_{p}\left(\boldsymbol{x} \times \boldsymbol{v}_{p}+\boldsymbol{\sigma}_{p}\right) v_{a}\right] \\
\frac{d}{d t} \sum \rho_{p} \boldsymbol{s}_{p} v_{a}=\sum\left(\sum_{n} \boldsymbol{m}_{p}^{(n)} a_{a}^{(n)}+\breve{b}_{p} \boldsymbol{s}_{p} v_{a}\right) \\
\frac{d}{d t} \sum \rho_{p}\left(\varepsilon_{p}+\frac{1}{2} \boldsymbol{v}_{p} \cdot \boldsymbol{v}_{p}+\frac{1}{2} \boldsymbol{s}_{p} \cdot \boldsymbol{\omega}_{p}\right) v_{a} \\
=\sum\left[\sum_{n}\left(\boldsymbol{t}_{p}^{(n)} \cdot \boldsymbol{v}_{p}+\boldsymbol{m}_{p}^{(n)} \cdot \boldsymbol{\omega}_{p}+q_{p}^{(n)}\right) a_{a}^{(n)}+\left(\boldsymbol{v}_{p} \cdot \breve{\boldsymbol{p}}_{p}+\boldsymbol{\omega}_{p} \cdot \breve{\boldsymbol{l}}_{p}+\breve{\varepsilon}_{p}\right) v_{a}+\breve{b}_{p}\left(\varepsilon_{p}+\frac{1}{2} \boldsymbol{v}_{p} \cdot \boldsymbol{v}_{p}+\frac{1}{2} \boldsymbol{s}_{p} \cdot \boldsymbol{\omega}_{p}\right) v_{a}\right]
\end{gathered}
$$

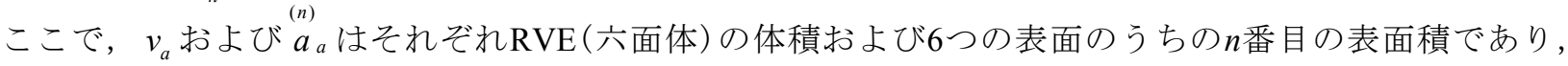
$\sum$ はRVE内での平均値の検査体積 $v$ わたる和を表わしている. また $\rho_{p}, \boldsymbol{v}_{p}, \boldsymbol{\sigma}_{p}, \boldsymbol{s}_{p}, \boldsymbol{\varepsilon}_{p}$ および $\boldsymbol{\omega}_{p}$ は, それぞれRVE内で平均化された相 $p$ の密度, 速度, 単位質量あたりの公転角運動量, 単位質量あたりのスピ ン角運動量, 内部エネルギーおよびスピン角速度であり， $\boldsymbol{\sigma}_{p}, \boldsymbol{n}_{p}$ および $\breve{\boldsymbol{\mu}}_{p}$ はそれぞれ次式のように定義 されている(25).

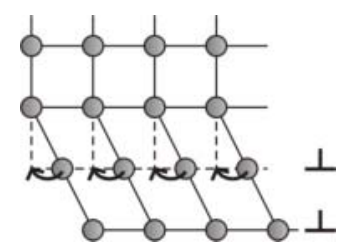

Fig. 1 Rotation of lattice

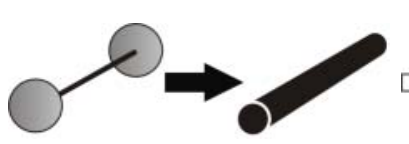

Fig. 2 Lattice element

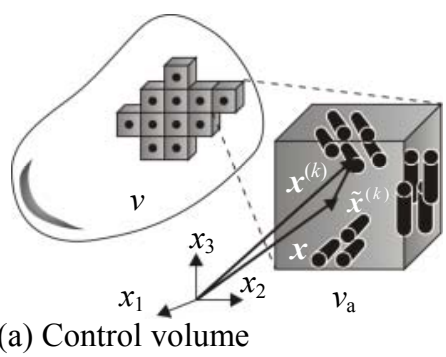

(b) Representative volume element
Fig. 3 Couple acting on lattice element 


$$
\boldsymbol{\sigma}_{p} \equiv \frac{1}{m_{a p}} \sum_{k=1}^{N_{v p}} m_{p}^{(k)} \tilde{\boldsymbol{x}}^{(k)} \times \tilde{\boldsymbol{v}}_{p}^{(k)}, \stackrel{(n)}{\boldsymbol{n}_{p}} \equiv \frac{1}{a_{a_{a}}} \sum_{l=1}^{\substack{(n) \\ N_{a p}}} \tilde{\boldsymbol{x}}^{(l)} \times \boldsymbol{F}_{p}^{(l)}, \breve{\boldsymbol{\mu}}_{p} \equiv \frac{1}{v_{a}} \sum_{k=1}^{N_{v p}} \tilde{\boldsymbol{x}}^{(k)} \times \breve{\boldsymbol{P}}_{p}^{(k)}
$$

ここで， $m_{a p}$ はRVE内の相 $p$ の全質量， $k$ および $N_{v p}$ はRVE内にある格子要素の番号およびその個数， $l$ およ び $N_{a p}$ はそれぞれ $\stackrel{(n)}{a}_{a}$ 面上にある格子要素の番号およびその要素数である.また, $m_{p}^{(k)}, \tilde{\boldsymbol{x}}^{(k)}, \tilde{\boldsymbol{v}}_{p}^{(k)}$ および $\boldsymbol{F}_{p}^{(k)} \equiv \boldsymbol{F}_{1 p}^{(k)}+\boldsymbol{F}_{2 p}^{(k)}$ はそれぞれ相 $p$ における $k$ 番目の格子要素の質量, RVEおよび格子要素の重心を結ぶべク ト $_{(n)}\left(\right.$ 図4)，同ベクトルの速度および格子要素の両端に働く原子間力の重心等価力(図3)であり， $\boldsymbol{t}_{p}, \quad \boldsymbol{n}_{p}$, $\boldsymbol{m}_{p}$ および $q_{p}$ はそれぞれ $n$ 番目の表面積に働く表面力, 公転のための表面偶応力, スピンのための表面偶 応力および単位時間あたりに同表面から供給される単位面積あたりの熱である. 一方, $\breve{b}_{p}, \breve{\boldsymbol{p}}_{p}, \breve{\boldsymbol{\mu}}_{p}, \breve{\boldsymbol{l}}_{p}$ および $\breve{\varepsilon}_{p}$ はそれぞれRVE内で平均化された質量湧き出し, 相互作用運動量, 格子のモーメントアーム $\tilde{\boldsymbol{x}}^{(k)}$ と相互作用運動量 $\breve{\boldsymbol{p}}_{p}^{(k)}$ が形成する相互作用角運動量, 相互作用角運動量および相互作用エネルギーである (25).

\section{3. 相 $p$ に対する連続体としての保存則（全体形および局所形）}

\section{$3 \cdot 1$ 単相における運動学および輸送定理}

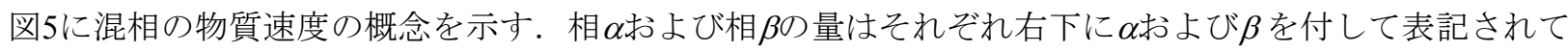
いる. 図5のように物質の参照配置における位置ベクトルを $\boldsymbol{X}_{p}$ および現配置での位置ベクトルを $\boldsymbol{x}$ とおく と，現配置における相 $p$ の物質速度は次式のように書ける。

$$
\boldsymbol{v}_{p} \equiv \frac{\bar{D} \boldsymbol{x}}{\bar{D} t} \equiv \grave{\boldsymbol{x}}\left(\boldsymbol{X}_{p}, t\right)=\left(\frac{\partial \boldsymbol{x}\left(\boldsymbol{X}_{p}, t\right)}{\partial t}\right)_{\boldsymbol{X}_{p}}
$$

以降, 任意の量 $A$ の単相に対する物質時間微分を $\dot{A}$ と書く ${ }^{(24)}$. 微小線素 $d \boldsymbol{x}$ は変形こう配テンソル $\boldsymbol{F}_{p}=\partial \boldsymbol{x} / \partial \boldsymbol{X}_{p}$ を用いて表すと, 次式のようになる.

$$
d \boldsymbol{x}=\boldsymbol{F}_{p} d \boldsymbol{X}_{p}
$$

式(8)より $d \boldsymbol{x}$ のスカラー三重積を作れば微小体積要素 $d v$ は次式のように書ける.

$$
d v=J_{p} d v_{0 p}
$$

ここで, $J_{p}$ は相 $p$ のコビアンであり, 次式のように定義される.

$$
J_{p} \equiv \operatorname{det} \boldsymbol{F}_{p}
$$

式(10)に単相の物質時間微分を施せば, $\grave{\boldsymbol{F}}_{p}=\left(\operatorname{grad} \boldsymbol{v}_{p}\right) \boldsymbol{F}_{p}$ を考慮して $\dot{J}_{p}$ が

$$
\grave{J}_{p}=J_{p} \operatorname{div} \boldsymbol{v}_{p}
$$

のように得られる. また, 相 $p$ の任意の量 $A_{p}$ に対する単相の物質時間微分は以下のように定義される(24).

$$
\frac{\bar{D} A_{p}}{\bar{D} t} \equiv \dot{A}_{p} \equiv \frac{\partial A_{p}}{\partial t}+\left(\boldsymbol{v}_{p} \cdot \operatorname{grad}\right) A_{p}
$$

一方, 通常の物質時間微分は次式で定義される.

$$
\frac{D A_{p}}{D t} \equiv \dot{A}_{p} \equiv \frac{\partial A_{p}}{\partial t}+(\boldsymbol{v} \cdot \operatorname{grad}) A_{p}
$$

式(9)，(11)および式(12)より， $A_{p}$ に対する，単相の輸送定理(対流形表示)が次式のように導ける.

$$
\frac{\bar{D}}{\bar{D} t} \int_{\mathrm{V}} A_{p} d v=\int_{\mathrm{V}}\left(\dot{A}_{p}+A_{p} \operatorname{div} \boldsymbol{v}_{p}\right) d v
$$

また，式(12)を式(14)に適用すれば，輸送定理は次式のように保存形表示へと変更される.

$$
\frac{\bar{D}}{\bar{D} t} \int_{\mathrm{V}} A_{p} d v=\int_{\mathrm{V}}\left[\frac{\partial A_{p}}{\partial t}+\operatorname{div}\left(A_{p} \boldsymbol{v}_{p}\right)\right] d v
$$

\section{$3 \cdot 2$ 質量保存則}

式(1)で $v_{a} \rightarrow d v$ のように極限をとって，RVEを連続体の一物質点に帰着させれば，相 $p$ に対する連続体と しての質量保存則が 


$$
\frac{\bar{D}}{\bar{D} t} \int_{\vee} \rho_{p} d v=\int_{\vee} \breve{b}_{p} d v
$$

のように得られる. 式(1)では， $\rho_{p}(\boldsymbol{x}, t)$ における $\boldsymbol{x}$ は図4に示寸とおりvにおける各RVEの重心の位置であ るから離散量であり, 時間の常微分を用いて表記される. しかし, RVEが物質点に収束することによって $\boldsymbol{x}$ は連続量となるため, 式(16)は単相の物質時間微分を用いて表わされる. さらに, 式(16)左辺に式(14)を 適用し，局所化すれば，相 $p$ にける連続の式が

$$
\grave{\rho}_{p}+\rho_{p} \operatorname{div} \boldsymbol{v}_{p}=\breve{b}_{p}
$$

のように得られる．式(12)を式(17)に用いれば，次式のようになる.

$$
\frac{\partial \rho_{p}}{\partial t}=-\operatorname{div}\left(\rho_{p} \boldsymbol{v}_{p}\right)+\breve{b}_{p}
$$

さらに， $A_{p}$ が $A_{p}=\rho_{p} a_{p}$ と書けるときには，式(9)および式(11)から

$$
\begin{gathered}
\frac{\bar{D}}{\bar{D} t} \int_{V} \rho_{p} a_{p} d v=\frac{\bar{D}}{\bar{D} t} \int_{V} \rho_{p} a_{p} J_{p} d v_{0 p}=\int_{V}\left(\rho_{p} a_{p} J_{p}\right) \dot{d} v_{0_{p}}=\int_{V}\left(\grave{\rho}_{p} a_{p} J_{p}+\rho_{p} \grave{a}_{p} J_{p}+\rho_{p} a_{p} \grave{J}_{p}\right) d v_{0 p} \\
=\int_{V}\left(\grave{\rho}_{p} a_{p}+\rho_{p} \grave{a}_{p}+\rho_{p} a_{p} \operatorname{div} \boldsymbol{v}_{p}\right) J_{p} d v_{0 p}=\int_{V}\left\{\rho_{p} \grave{a}_{p}+a_{p}\left(\grave{\rho}_{p}+\rho_{p} \operatorname{div} \boldsymbol{v}_{p}\right)\right\} d v
\end{gathered}
$$

なる関係式が得られる. 式(17)を式(19)に代入すれば， $\rho_{p} a_{p}$ に対する輸送定理が次式のように得られる.

$$
\frac{\bar{D}}{\bar{D} t} \int_{V} \rho_{p} a_{p} d v=\int_{\vee}\left(\rho_{p} \grave{a}_{p}+\breve{b}_{p} a_{p}\right) d v
$$

\section{$3 \cdot 3$ 運動量保存則}

式(2)において, $v_{a} \rightarrow d v$ および $a_{a} \rightarrow d a$ の極限操作を行い, RVEを連続体の一物質点に帰着させれば

$$
\frac{\bar{D}}{\bar{D} t} \int_{V} \rho_{p} \boldsymbol{v}_{p} d v=\oint_{\mathrm{S}}^{\boldsymbol{t}_{p}} d a+\int_{V}\left(\breve{\boldsymbol{p}}_{p}+\breve{b}_{p} \boldsymbol{v}_{p}\right) d v
$$

を得る.式(21)は，相 $p$ に対する連続体としての運動量保存則を表す．さらに式(20)を式(21)左辺に適用すれ ば次式のようになる.

$$
\frac{\bar{D}}{\bar{D} t} \int_{\vee} \rho_{p} \boldsymbol{v}_{p} d v=\int_{\vee}\left(\rho_{p} \dot{\boldsymbol{v}}_{p}+\breve{b}_{p} \boldsymbol{v}_{p}\right) d v
$$

また, 式(21)右辺第1項に, Cauchyの基本定理 $\boldsymbol{t}_{p}^{(n)} \equiv \boldsymbol{T}_{p} \boldsymbol{n}$ およびGaussの発散定理を適用すれば, 次式のように なる.

$$
\oint_{\mathrm{S}}^{\left(\boldsymbol{t}_{p}\right)} d a=\oint_{\mathrm{S}} \boldsymbol{T}_{p} \boldsymbol{n} d a=\int_{\mathrm{V}} \operatorname{div} \boldsymbol{T}_{p} d v
$$

式(22)および式(23)を式(21)に代入するとともに局所化して整理すれば，次式が得られる.

$$
\rho_{p} \dot{\boldsymbol{v}}_{p}=\operatorname{div} \boldsymbol{T}_{p}+\breve{\boldsymbol{p}}_{p}
$$

\section{$3 \cdot 4$ 角運動量保存則}

\section{$3 \cdot 4 \cdot 1$ バルクの角運動量保存則}

式(3)で $v_{a} \rightarrow d v$ および $a_{a} \rightarrow d a$ とすれば，図4よりRVEの重心が物質点へと帰着し， $\tilde{\boldsymbol{x}}^{(k)} \rightarrow 0$ となるため, 式(6)より $\boldsymbol{\sigma}_{p} \rightarrow 0, \boldsymbol{n}_{p} \rightarrow 0$ および $\breve{\boldsymbol{\mu}}_{p} \rightarrow 0$ となる. したがって, 式(3)についてRVEを連続体の一物質点に帰

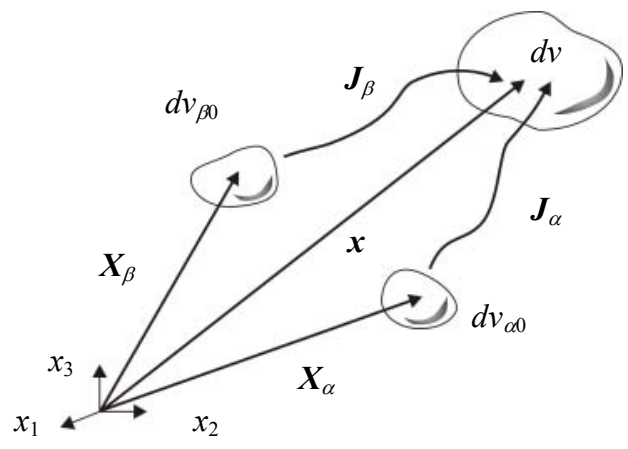

Fig. 5 Mixture model 
着させれば,

$$
\frac{\bar{D}}{\bar{D} t} \int_{\vee} \rho_{p} \boldsymbol{x} \times \boldsymbol{v}_{p} d v=\oint_{\mathrm{S}} \boldsymbol{x} \times \stackrel{(n)}{p}_{p}^{(n)} d a+\int_{\vee}\left[\left(\boldsymbol{x} \times \breve{\boldsymbol{p}}_{p}+\breve{\boldsymbol{l}}_{p}\right)+\breve{b}_{p} \boldsymbol{x} \times \boldsymbol{v}_{p}\right] d v
$$

が得られる. 式(25)は相 $p$ に対する連続体としてのバルクの角運動量保存則であり， $\tilde{\boldsymbol{x}}^{(k)}$ に起因する相関 $(2$ 次のゆらぎの平均) はもはや現れない.さらに，式(7)および式(20)を式(25)左辺に適用すれば次式のように なる．ただし， $\dot{\boldsymbol{x}} \times \boldsymbol{v}_{p}=\boldsymbol{v}_{p} \times \boldsymbol{v}_{p}=\mathbf{0}$ が考慮されている.

$$
\frac{\bar{D}}{\bar{D} t} \int_{\vee} \rho_{p} \boldsymbol{x} \times \boldsymbol{v}_{p} d v=\int_{\mathrm{V}}\left(\rho_{p} \boldsymbol{x} \times \dot{\boldsymbol{v}}_{p}+\boldsymbol{x} \times \breve{b}_{p} \boldsymbol{v}_{p}\right) d v
$$

また, 式(25)右辺第1項に, Cauchyの基本定理およびGaussの発散定理を適用すれば，次式のようになる.

$$
\oint_{\mathrm{S}} \boldsymbol{x} \times \stackrel{(n)}{p}_{p} d a=\oint_{\mathrm{S}} \boldsymbol{x} \times \boldsymbol{T}_{p} \boldsymbol{n} d a=\int_{V}\left[\boldsymbol{e} \cdot \boldsymbol{T}_{p}+\boldsymbol{x} \times\left(\operatorname{div} \boldsymbol{T}_{p}\right)\right] d v
$$

ここで, $\boldsymbol{e} \cdot \cdot \boldsymbol{T}_{p}=e_{i j k} T_{k j}^{p} \boldsymbol{e}_{i}$ であり， $\boldsymbol{e}_{i}$ は基底ベクトルである．なお， $T_{i j}^{p}$ のようにテンソル成分においては相 番号 $p$ を上添字で表記する. 式(26)および式(27)を式(25)に代入して局所化するとともに, 式(24)を考慮して 整理すれば，次式のようになる.

\section{$3 \cdot 4 \cdot 2$ 格子の角運動量保存則}

$$
\boldsymbol{e} \cdot \boldsymbol{T}_{p}+\breve{\boldsymbol{l}}_{p}=0
$$

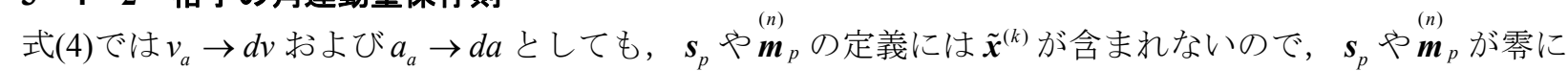
収束することはない。このことは，連続体の物質点に格子要素のスピン角運動量(あるいは方位) 情報が付 随することを意味しており, そのような付随ベクトルは文献(22)のディレクタに相当するものとみなせる. したがって，式(4)についてRVEを連続体の一物質点に帰着させれば次式が得られる.

$$
\frac{\bar{D}}{\bar{D} t} \int_{\vee} \rho_{p} \boldsymbol{s}_{p} d v=\oint_{\mathrm{S}} \boldsymbol{m}_{p}^{(n)} d a+\int_{\vee} \breve{b}_{p} \boldsymbol{s}_{p} d v
$$

式(29)は，相 $p$ に対する連続体としての格子要素の角運動量保存則である. 式(20)を式(29)左辺に適用すれば 次式のようになる.

$$
\frac{\bar{D}}{\bar{D} t} \int_{\vee} \rho_{p} \boldsymbol{s}_{p} d v=\int_{\mathrm{V}}\left(\rho_{p} \grave{\boldsymbol{s}}_{p}+\breve{b}_{p} \boldsymbol{s}_{p}\right) d v
$$

また, 式(29)右辺第1項に, 偶応力に対するCauchyの基本定理 $\boldsymbol{m}_{p}{ }^{(n)} \boldsymbol{M}_{p} \boldsymbol{n}$ およびGaussの発散定理を適用すれ ば, 次式のようになる.

$$
\oint_{\mathrm{S}} \boldsymbol{m}_{p}^{(n)} d a=\oint_{\mathrm{S}} \boldsymbol{M}_{p} \boldsymbol{n} d a=\int_{\mathrm{V}} \operatorname{div} \boldsymbol{M}_{p} d v
$$

式(30)および式(31)を式(29)に代入するとともに，局所化して整理すれば，次式を得る.

$$
\rho_{p} \grave{s}_{p}=\operatorname{div} \boldsymbol{M}_{p}
$$

\section{$3 \cdot 5$ エネルギー保存則}

式(5)において $v_{a} \rightarrow d v$ および $a_{a} \rightarrow d a$ とし, RVEを連続体の一物質点に帰着させれば次のようになる.

$$
\begin{gathered}
\frac{\bar{D}}{\bar{D} t} \int_{V} \rho_{p}\left(\varepsilon_{p}+\frac{1}{2} \boldsymbol{v}_{p} \cdot \boldsymbol{v}_{p}+\frac{1}{2} \boldsymbol{s}_{p} \cdot \boldsymbol{\omega}_{p}\right) d v \\
\left.=\oint_{\mathrm{S}}\left(\boldsymbol{t}_{p}^{(n)} \cdot \boldsymbol{v}_{p}+\stackrel{\boldsymbol{m}}{p}_{p} \cdot \boldsymbol{\omega}_{p}+\stackrel{(n)}{p}\right)^{\prime}\right) d a+\int_{V}\left\{\left(\boldsymbol{v}_{p} \cdot \breve{\boldsymbol{p}}_{p}+\boldsymbol{\omega}_{p} \cdot \breve{\boldsymbol{l}}_{p}+\breve{\varepsilon}_{p}\right)+\breve{b}_{p}\left(\varepsilon_{p}+\frac{1}{2} \boldsymbol{v}_{p} \cdot \boldsymbol{v}_{p}+\frac{1}{2} \boldsymbol{s}_{p} \cdot \boldsymbol{\omega}_{p}\right)\right\} d v
\end{gathered}
$$

式(33)は，相 $p$ に対する連続体としてのエネルギー保存則を表す．またこのとき， $3 \cdot 4 ・ 1$ 項と同様に $\tilde{\boldsymbol{x}}^{(k)} \rightarrow 0$ となるのにともなって, $\varepsilon_{p}$ および $q_{p}$ に陰に内在していたRVE内での格子要素の公転による角運 動量の項も消失する. 式(14)を式(33)左辺に適用すれば次式のようになる.

$$
\frac{\bar{D}}{\bar{D} t} \int_{V} \rho_{p} e_{p} d v=\int_{V}\left\{\left(\rho_{p} \dot{\varepsilon}_{p}+\breve{b}_{p} \varepsilon_{p}\right)+\frac{1}{2}\left[\rho_{p}\left(\boldsymbol{v}_{p} \cdot \boldsymbol{v}_{p}\right)+\breve{b}_{p} \boldsymbol{v}_{p} \cdot \boldsymbol{v}_{p}\right]+\frac{1}{2}\left[\rho_{p}\left(\boldsymbol{s}_{p} \cdot \boldsymbol{\omega}_{p}\right)+\breve{b}_{p} \boldsymbol{v}_{p} \cdot \boldsymbol{v}_{p}\right]\right\} d v
$$

ここで， $e_{p}$ は次式で定義される相 $p$ の全エネルギー密度である.

$$
e_{p} \equiv \varepsilon_{p}+\frac{1}{2} \boldsymbol{v}_{p} \cdot \boldsymbol{v}_{p}+\frac{1}{2} \boldsymbol{s}_{p} \cdot \boldsymbol{\omega}_{p}
$$


また, 式(33)右辺最初の積分項に, Cauchyの基本定理, Stokesの熱流束定理 $\stackrel{(n)}{p}_{p}=-\boldsymbol{q}_{p} \cdot \boldsymbol{n}$ およびGaussの発散 定理を適用すれば,

$$
\oint_{\mathrm{S}}\left(\boldsymbol{t}_{p}^{(n)} \cdot \boldsymbol{v}_{p}+\boldsymbol{m}_{p}^{(n)} \cdot \boldsymbol{\omega}_{p}+{ }_{q}^{(n)}\right) d a=\oint_{\mathrm{S}}\left(\boldsymbol{T}_{p}^{T} \boldsymbol{v}_{p}+\boldsymbol{M}_{p}^{T} \boldsymbol{\omega}_{p}+\boldsymbol{q}_{p}\right) \cdot \boldsymbol{n} d a=\int_{\mathrm{V}} \operatorname{div}\left(\boldsymbol{T}_{p}^{T} \boldsymbol{v}_{p}+\boldsymbol{M}_{p}^{T} \boldsymbol{\omega}_{p}-\boldsymbol{q}_{p}\right) d v
$$

のようになる. 式(34)および式(36)を式(33)に代入して，局所化して整理すれば，次式を得る.

$$
\rho_{p} \grave{e}_{p}=-\operatorname{div}\left(\boldsymbol{q}_{p}-\boldsymbol{T}_{p}^{T} \boldsymbol{v}_{p}-\boldsymbol{M}_{p}^{T} \boldsymbol{\omega}_{p}\right)+\boldsymbol{v}_{p} \cdot \breve{\boldsymbol{p}}_{p}+\boldsymbol{\omega}_{p} \cdot \breve{\boldsymbol{l}}_{p}+\breve{\varepsilon}_{p}
$$

\section{4. 混相に対する保存則}

\section{$4 \cdot 1$ 混相のための平均化および非定常項に対する定理}

混相における物理量として，ここでは原子レベルのゆらぎの表記と混相における相対量の表記を区別す るために, 後者を $\bar{A}_{p}$ のように書き, $A_{p}$ を

$$
A_{p} \equiv A+\widehat{A}_{p}
$$

のように分離する．ただし， $A$ は混相における平均量である．各相の密度の和は混相の密度になるから，

$$
\rho=\sum_{p} \rho_{p}
$$

である．また，質量平均の定義式

$$
A \equiv \frac{1}{\rho} \sum_{p} \rho_{p} A_{p}
$$

より，次式が成立する.

$$
A=\frac{1}{\rho} \sum_{p} \rho_{p} A_{p}=\frac{1}{\rho} \sum_{p} \rho_{p}\left(A+\widehat{A}_{p}\right)=\frac{1}{\rho}\left(\sum_{p} \rho_{p}\right) A+\frac{1}{\rho} \sum_{p} \rho_{p} \widehat{A}_{p}=A+\frac{1}{\rho} \sum_{p} \rho_{p} \widehat{A}_{p}
$$

式(41)を式(40)と比較することにより相対量の質量平均の和が零になることがわかる.

$$
\sum_{p} \rho_{p} \widehat{A}_{p}=0
$$

さらに, 反応(ここでは相転移)による質量湧き出しの和は零となるから, 次式が成り立つ(24).

$$
\sum_{p} \breve{b}_{p}=0
$$

一方, 混相の保存則は単純体の保存則に一致しなくてはならないから, 最終的に得られる保存則では相互 作用 $\breve{A}_{p}$ および質量涌き出しによる相互作用 $\breve{b}_{p} B_{p}$ の和として表される全相互作用は零である(24). ただし， 後述するように, 保存則の中には $\breve{A}_{p}$ あるいは $\breve{b}_{p} B_{p}$ のいずれか一つの項しか存在しないものもある.

$$
\sum_{p}\left(\breve{A}_{p}-\breve{b}_{p} B_{p}\right)=0
$$

次に，式(38)を非定常項の混合和 $\sum_{p} \rho_{p} \grave{A}_{p}$ に適用すれば

$$
\sum_{p} \rho_{p} \grave{A}_{p}=\sum_{p} \rho_{p}\left(\grave{A}+\grave{A}_{p}\right)
$$

のようになる。ここで，式(12)，(38)および式(42)を式(45)右辺（）内第1項に用いれば，次式のようになる.

$$
\sum_{p} \rho_{p} \dot{A}=\sum_{p} \rho_{p}\left\{\frac{\partial A}{\partial t}+\left[\left(\boldsymbol{v}+\widehat{\boldsymbol{v}}_{p}\right) \cdot \operatorname{grad}\right] A\right\}=\left[\frac{\partial A}{\partial t}+(\boldsymbol{v} \cdot \mathrm{grad}) A\right] \sum_{p} \rho_{p}+\left[\left(\sum_{p} \rho_{p} \widehat{\boldsymbol{v}}_{p}\right) \cdot \operatorname{grad}\right] A=\rho \dot{A}
$$

また，式(45)右辺( )内第2項は式(12)および式(18)を用いて

$$
\begin{gathered}
\sum_{p} \rho_{p} \grave{A}_{p}=\sum_{p} \rho_{p}\left[\frac{\partial \widehat{A}_{p}}{\partial t}+\left(\boldsymbol{v}_{p} \cdot \operatorname{grad}\right) \widehat{A}_{p}\right]=\sum_{p}\left[\frac{\partial \rho_{p} \widehat{A}_{p}}{\partial t}-\widehat{A}_{p} \frac{\partial \rho_{p}}{\partial t}+\rho_{p}\left(\boldsymbol{v}_{p} \cdot \operatorname{grad}\right) \widehat{A}_{p}\right] \\
=\sum_{p}\left[\frac{\partial \rho_{p} \widehat{A}_{p}}{\partial t}+\widehat{A}_{p} \operatorname{div}\left(\rho_{p} \boldsymbol{v}_{p}\right)-\breve{b}_{p} \widehat{A}_{p}+\rho_{p}\left(\boldsymbol{v}_{p} \cdot \operatorname{grad}\right) \widehat{A}_{p}\right]=\sum_{p}\left[\frac{\partial \rho_{p} \widehat{A}_{p}}{\partial t}+\operatorname{div}\left(\widehat{A}_{p} \rho_{p} \boldsymbol{v}_{p}\right)-\breve{b}_{p} \widehat{A}_{p}\right]
\end{gathered}
$$

となる．このとき式(47)右辺 [ ]内第1項は

$$
\sum_{p} \frac{\partial \rho_{p} \bar{A}_{p}}{\partial t}=\frac{\partial}{\partial t} \sum_{p}\left(\rho_{p} \bar{A}_{p}\right)=0
$$


である．また，式(47)右辺 [ ]内第2項は，式(38)および式(42)を考慮して，

$$
\sum_{p} \operatorname{div}\left(\widehat{A}_{p} \rho_{p} \boldsymbol{v}_{p}\right)=\operatorname{div} \sum_{p}\left[\widehat{A}_{p} \rho_{p}\left(\boldsymbol{v}+\widehat{\boldsymbol{v}}_{p}\right)\right]=\operatorname{div}\left(\boldsymbol{v} \sum_{p} \rho_{p} \widehat{A}_{p}+\sum_{p} \rho_{p} \widehat{A}_{p} \widehat{\boldsymbol{v}}_{p}\right)=\operatorname{div} \sum_{p} \rho_{p} \widehat{A}_{p} \widehat{\boldsymbol{v}}_{p}=\operatorname{div} \sum_{p} \rho_{p} A_{p} \widehat{\boldsymbol{v}}_{p}
$$

のように書ける，さらに，式(47)右辺 [ ]内第3項は式(43)より

$$
-\sum_{p} \breve{b}_{p} \widehat{A}_{p}=-\sum_{p} \breve{b}_{p}\left(A-A_{p}\right)=-A \sum_{p} \breve{b}_{p}+\sum_{p} \breve{b}_{p} A_{p}=\sum_{p} \breve{b}_{p} A_{p}
$$

となる. 式(46)〜 (50)を式(45)に代入すれば，次のような非定常項の混合に対する定理が得られる.

$$
\sum_{p} \rho_{p} \grave{A}_{p}=\rho \dot{A}+\operatorname{div} \sum_{p} \rho_{p} A_{p} \widehat{\boldsymbol{v}}_{p}+\sum_{p} \breve{b}_{p} A_{p}
$$

\section{$4 \cdot 2$ 質量保存則}

式(38)を式(18)の $\boldsymbol{v}_{p}$ に適用すれば,

$$
\frac{\partial \rho_{p}}{\partial t}+\left(\operatorname{grad} \rho_{p}\right) \cdot \boldsymbol{v}+\rho_{p} \operatorname{div} \boldsymbol{v}+\operatorname{div}\left(\rho_{p} \widehat{\boldsymbol{v}}_{p}\right)-\breve{b}_{p}=0
$$

のように書き改められる．式(52)の両辺を相にわたって和をとれば，次式のようになる.

$$
\sum_{p}\left[\frac{\partial \rho_{p}}{\partial t}+\boldsymbol{v} \cdot\left(\operatorname{grad} \rho_{p}\right)+\rho_{p} \operatorname{div} \boldsymbol{v}+\operatorname{div}\left(\rho_{p} \widehat{\boldsymbol{v}}_{p}\right)\right]-\sum_{p} \breve{b}_{p}=0
$$

ここで，式(39)，(42)および式(43)より，式(53)は

$$
\frac{\partial \rho}{\partial t}+(\operatorname{grad} \rho) \cdot \boldsymbol{v}+\rho \operatorname{div} \boldsymbol{v}=0
$$

となる，通常の物質時間微分の定義から，式(54)は次式のように書き改められる.

$$
\dot{\rho}+\rho \operatorname{div} \boldsymbol{v}=0
$$

式(55)は連続の式であり, 混相の質量保存則は単純体の質量保存則と形式的に一致することがわかる.

一方，式(13)を式(52)に用い，通常の物質時間微分で表記すれば,

$$
\dot{\rho}_{p}+\rho_{p} \operatorname{div} \boldsymbol{v}+\operatorname{div}\left(\rho_{p} \widehat{\boldsymbol{v}}_{p}\right)-\breve{b}_{p}=0
$$

が得られる.さらに相対質量流束 $\boldsymbol{j}_{p}$ を

$$
\boldsymbol{j}_{p} \equiv \rho_{p} \widehat{\boldsymbol{v}}_{p}
$$

のように定義すれば, 相 $p$ にける質量保存則が次式のように書き改められる.

$$
\dot{\rho}_{p}+\rho_{p} \operatorname{div} \boldsymbol{v}+\operatorname{div} \boldsymbol{j}_{p}-\breve{b}_{p}=0
$$

式(58)の保存則は, 複数相が存在するときの一つの相であればどのような相に対しても成り立つ. 再結晶 現象のように転位密度の差によって母相と再結晶相の物性が異なっている場合でも, 保存則は物質によら ない一般原理であるため, 通常と同様, 両相に対して同じ形式で書かれなければならない. 各相に含まれ る転位密度の相違などの材料特性は流束の構成式や質量湧き出し項の具体化において表現される.また, 再結晶を考える場合には, 相 $p$ を相 $(\phi=0)$ および再結晶相 $(\phi=1)$ のみに限定し, 秩序変数 $\phi$ を再結晶相 $(p$ $=r)$ の体積分率と定義すればよい，このとき再結晶相の密度 $\rho_{r}$ は $\rho_{r}=\rho \phi$ と表わされる. これを式(58)に代 入し，式(55)を用いれば，次式のように秩序変数 $\phi$ の釣合い方程式が得られる．ただし，ここでは静的再結 晶のみを考え，母相，再結晶相ともに密度は一定の $\rho$ としている.

$$
\dot{\phi}=-\operatorname{div} \boldsymbol{j}_{\phi}+b_{\phi}
$$

ここで， $\boldsymbol{j}_{\phi}$ および $b_{\phi}$ はそれぞれ $\boldsymbol{j}_{\phi} \equiv \overline{\boldsymbol{j}}_{r} / \rho$ および $b_{\phi} \equiv \breve{b}_{r} / \rho$ である．秩序流束 $\boldsymbol{j}_{\phi}$ の構成式が導出され，相転 移による $\phi$ の湧き出し $b_{\phi}$ が具体的にモデル化されれば，再結晶に対寸るPhase-fieldモデルにおける $\phi$ 支配 方程式が導出されることが予想される.

\section{$4 \cdot 3$ 運動量保存則}

式(24)を相にわたって和をとれば，次式のようになる.

$$
\sum_{p} \rho_{p} \dot{\boldsymbol{v}}_{p}=\sum_{p} \operatorname{div} \boldsymbol{T}_{p}+\sum_{p} \breve{\boldsymbol{p}}_{p}
$$

ここで，式(51)を式(60)左辺に適用し，式(42)を考慮すれば 


$$
\sum_{p} \rho_{p} \grave{\boldsymbol{v}}_{p}=\rho \dot{\boldsymbol{v}}+\operatorname{div} \sum_{p} \rho_{p} \widehat{\boldsymbol{v}}_{p} \otimes \widehat{\boldsymbol{v}}_{p}+\sum_{p} \breve{b}_{p} \boldsymbol{v}_{p}
$$

となる，式(61)を式(60)に代入すれば，次式を得る.

$$
\rho \dot{\boldsymbol{v}}=\operatorname{div} \sum_{p}\left(\boldsymbol{T}_{p}-\rho_{p} \widehat{\boldsymbol{v}}_{p} \otimes \widehat{\boldsymbol{v}}_{p}\right)+\sum_{p}\left(\breve{\boldsymbol{p}}_{p}-\breve{b}_{p} \boldsymbol{v}_{p}\right)
$$

ここで, 混相の応力 $\boldsymbol{T}$ を

$$
\boldsymbol{T} \equiv \boldsymbol{T}_{\mathrm{I}}-\sum_{p} \rho_{p} \widehat{\boldsymbol{v}}_{p} \otimes \widehat{\boldsymbol{v}}_{p}
$$

のように定義する ${ }^{(24)}$. ただし， $\boldsymbol{T}_{\mathrm{I}}$ は各相の応力の単純和であり，

$$
\boldsymbol{T}_{\mathrm{I}} \equiv \sum_{p} \boldsymbol{T}_{p}
$$

のように定義される，さらに，式(44)を式(62)右辺第2項に適用すれば次式のようになる.

$$
\sum_{p}\left(\breve{\boldsymbol{p}}_{p}-\breve{b}_{p} \boldsymbol{v}_{p}\right)=0
$$

式(63)および式(65)を用いれば，式(62)は通常の運動方程式の形式となる.

$$
\rho \dot{\boldsymbol{v}}=\operatorname{div} \boldsymbol{T}
$$

\section{$4 \cdot 4$ 角運動量保存則}

\section{$4 \cdot 4 \cdot 1$ バルクの角運動量保存則}

式(28)を相にわたって和をとり，式(64)を用いれば,

$$
\boldsymbol{e} \cdot \boldsymbol{T}_{\mathrm{I}}+\sum_{p} \breve{\boldsymbol{l}}_{p}=0
$$

のようになる．式(44)を式(67)左辺第2項に適用すれば,

$$
\sum_{p} \breve{\boldsymbol{l}}_{p}=0
$$

であり，式(67)は

$$
\boldsymbol{e} \cdot \boldsymbol{T}_{\mathrm{I}}=0
$$

となる.したがって, 式(69)より $\boldsymbol{T}_{\mathrm{I}}^{T}=\boldsymbol{T}_{\mathrm{I}}$ であり， $\boldsymbol{T}_{\mathrm{I}}$ は対称テンソルであることがわかる.また， $\boldsymbol{T}_{\mathrm{I}}$ および $\rho_{p} \widehat{v}_{p} \otimes \widehat{\boldsymbol{v}}_{p}$ の対称性を考慮すれば，式(63)より

$$
\boldsymbol{T}=\boldsymbol{T}^{T}
$$

となり，これは応力テンソルが対称であることを示している．通常，マイクロポーラー理論 ${ }^{(20)(21)}$ のうな 偶応力が存在する理論体系においては応力には非対称性が発現する. しかしながら, 本理論では前報 ${ }^{(25)}$ ように角運動量保存則を格子のスケールとバルクのスケールに分離したことで, 応力の対称性が保たれて いることがわかる。

\section{$4 \cdot 4 \cdot 2$ 格子の角運動量保存則}

次に，式(32)を相にわたって和をとれば，次式のようになる.

$$
\sum_{p} \rho_{p} \grave{\boldsymbol{s}}_{p}=\sum_{p} \operatorname{div} \boldsymbol{M}_{p}
$$

ここで, 式(51)を式(71)左辺に適用し, 式(42)を考慮すれば,

$$
\sum_{p} \rho_{p} \grave{\boldsymbol{s}}_{p}=\rho_{p} \dot{\boldsymbol{s}}+\operatorname{div} \sum_{p} \rho_{p} \widehat{\boldsymbol{s}}_{p} \otimes \widehat{\boldsymbol{v}}_{p}+\sum_{p} \breve{b}_{p} \boldsymbol{s}_{p}
$$

となる. 式(72)を式(71)に代入すれば，次式のようになる.

$$
\rho \dot{\boldsymbol{s}}=\operatorname{div} \sum_{p}\left(\boldsymbol{M}_{p}-\rho_{p} \widehat{\boldsymbol{s}}_{p} \otimes \widehat{\boldsymbol{v}}_{p}\right)-\sum_{p} \breve{b}_{p} \boldsymbol{s}_{p}
$$

ここで, 混相における格子の偶応力 $\boldsymbol{M}$ および各相の単純和 $\boldsymbol{M}_{\mathrm{I}}$ を

$$
\boldsymbol{M} \equiv \boldsymbol{M}_{\mathrm{I}}-\sum_{p} \rho_{p} \widehat{\boldsymbol{s}}_{p} \otimes \widehat{\boldsymbol{v}}_{p}, \boldsymbol{M}_{\mathrm{I}} \equiv \sum_{p} \boldsymbol{M}_{p}
$$

のように定義する. また, 式(44)より式(73)右辺第2項は次式のようになる.

$$
\sum_{p} \breve{b}_{p} \boldsymbol{s}_{p}=0
$$

式(74)および式(75)を式(73)に代入すれば，格子の角運動量方程式が次式のように得られる. 


$$
\rho \dot{\boldsymbol{s}}=\operatorname{div} \boldsymbol{M}
$$

ここで, $\boldsymbol{s}$ は平均慣性モーメントテンソル $\boldsymbol{J}$ (付録参照)および平均スピン角速度 $\boldsymbol{\omega}=\dot{\boldsymbol{\theta}}$ を用いて

$$
\boldsymbol{s}=\boldsymbol{J} \omega, \omega=\dot{\theta}
$$

のように書ける.ここでも $\phi$ の場合と同様に, 式(77)を式(76)に代入すれば, Phase-fieldモデルにおける方 位方程式の両辺を時間で1回微分したものに相当する式が

$$
(\boldsymbol{J} \dot{\boldsymbol{\theta}})^{\cdot}=\operatorname{div} \boldsymbol{I}_{\theta}
$$

のように得られる。ここで， $\boldsymbol{I}_{\theta}$ は方位流束であり， $\boldsymbol{I}_{\theta} \equiv \boldsymbol{M} / \rho$ のように定義される. 式(78)の両辺を時間 で1回積分のうえ，方位流束の具体的な構成式を代入して係数をモデル化すれば，KWC形Phase-fieldモデル における方位の支配方程式が得られると考えられる.

\section{5 エネルギ一保存則}

式(37)を相にわたって和をとれば，次式のようになる.

$$
\sum_{p} \rho_{p} \grave{e}_{p}=-\sum_{p} \operatorname{div}\left(\boldsymbol{q}_{p}-\boldsymbol{T}_{p}^{T} \boldsymbol{v}_{p}-\boldsymbol{M}_{p}^{T} \boldsymbol{\omega}_{p}\right)+\sum_{p}\left(\boldsymbol{v}_{p} \cdot \breve{\boldsymbol{p}}_{p}+\boldsymbol{\omega}_{p} \cdot \breve{\boldsymbol{l}}_{p}+\breve{\boldsymbol{\varepsilon}}_{p}\right)
$$

ここで，式(51)を式(79)左辺に適用すれば次式のようになる.

$$
\sum_{p} \rho_{p} \grave{e}_{p}=\rho \dot{e}+\operatorname{div} \sum_{p} \rho_{p} e_{p} \widehat{\boldsymbol{v}}_{p}+\sum_{p} \breve{b}_{p} e_{p}
$$

式(35), (38), (39)および式(42)を考慮すれば, 式(80)右辺第2項における被微分量は次のように書き換えら れる。

$$
\begin{gathered}
\sum_{p} \rho_{p} e_{p} \widehat{\boldsymbol{v}}_{p}=\sum_{p} \rho_{p}\left(\varepsilon_{p}+\frac{1}{2} \boldsymbol{v}_{p} \cdot \boldsymbol{v}_{p}+\frac{1}{2} \boldsymbol{s}_{p} \cdot \boldsymbol{\omega}_{p}\right) \widehat{\boldsymbol{v}}_{p} \\
=\sum_{p} \rho_{p}\left[\varepsilon_{p}+\frac{1}{2}\left(\boldsymbol{v}+\widehat{\boldsymbol{v}}_{p}\right) \cdot\left(\boldsymbol{v}+\widehat{\boldsymbol{v}}_{p}\right)+\frac{1}{2}\left(\boldsymbol{s}+\widehat{\boldsymbol{s}}_{p}\right) \cdot\left(\boldsymbol{\omega}+\widehat{\boldsymbol{\omega}}_{p}\right)\right] \widehat{\boldsymbol{v}}_{p} \\
=\sum_{p} \rho_{p} \varepsilon_{p} \widehat{\boldsymbol{v}}_{p}+\frac{1}{2}\left[\boldsymbol{v} \cdot \boldsymbol{v} \sum_{p} \rho_{p} \widehat{\boldsymbol{v}}_{p}+2 \sum_{p}\left(\rho_{p} \boldsymbol{v} \cdot \widehat{\boldsymbol{v}}_{p}\right) \widehat{\boldsymbol{v}}_{p}+\sum_{p}\left(\rho_{p} \widehat{\boldsymbol{v}}_{p} \cdot \widehat{\boldsymbol{v}}_{p}\right) \widehat{\boldsymbol{v}}_{p}\right] \\
+\frac{1}{2}\left[\boldsymbol{s} \cdot \boldsymbol{\omega} \sum_{p} \rho_{p} \widehat{\boldsymbol{v}}_{p}+\sum_{p}\left(\rho_{p} \boldsymbol{s} \cdot \widehat{\boldsymbol{\omega}}_{p}\right) \widehat{\boldsymbol{v}}_{p}+\sum_{p}\left(\rho_{p} \widehat{\boldsymbol{s}}_{p} \cdot \boldsymbol{\omega}\right) \widehat{\boldsymbol{v}}_{p}+\sum_{p}\left(\rho_{p} \widehat{\boldsymbol{s}}_{p} \cdot \widehat{\boldsymbol{\omega}}_{p}\right) \widehat{\boldsymbol{v}}_{p}\right] \\
=\sum_{p} \rho_{p}\left(\varepsilon_{p}+\frac{1}{2} \widehat{\boldsymbol{v}}_{p} \cdot \widehat{\boldsymbol{v}}_{p}+\frac{1}{2} \widehat{\boldsymbol{s}}_{p} \cdot \widehat{\boldsymbol{\omega}}_{p}\right) \widehat{\boldsymbol{v}}_{p}+\sum_{p}\left(\rho_{p} \widehat{\boldsymbol{v}}_{p} \otimes \widehat{\boldsymbol{v}}_{p}\right)^{T} \boldsymbol{v}+\frac{1}{2} \sum_{p}\left(\rho_{p} \widehat{\boldsymbol{\omega}}_{p} \otimes \widehat{\boldsymbol{v}}_{p}\right)^{T} \boldsymbol{s}+\frac{1}{2} \sum_{p}\left(\rho_{p} \widehat{\boldsymbol{s}}_{p} \otimes \widehat{\boldsymbol{v}}_{p}\right)^{T} \boldsymbol{\omega}
\end{gathered}
$$

式(81)を式(80)に代入すれば，次式のようになる.

$$
\begin{gathered}
\sum_{p} \rho_{p} \grave{e}_{p}=\rho \dot{e}+\operatorname{div}\left[\sum_{p} \rho_{p}\left(\varepsilon_{p}+\frac{1}{2} \widehat{\boldsymbol{v}}_{p} \cdot \widehat{\boldsymbol{v}}_{p}+\frac{1}{2} \widehat{\boldsymbol{s}}_{p} \cdot \widehat{\boldsymbol{\omega}}_{p}\right) \widehat{\boldsymbol{v}}_{p}\right. \\
\left.+\sum_{p}\left(\rho_{p} \widehat{\boldsymbol{v}}_{p} \otimes \widehat{\boldsymbol{v}}_{p}\right)^{T} \boldsymbol{v}+\frac{1}{2}\left(\sum_{p}\left(\rho_{p} \widehat{\boldsymbol{\omega}}_{p} \otimes \widehat{\boldsymbol{v}}_{p}\right)^{T} \boldsymbol{s}+\sum_{p}\left(\rho_{p} \widehat{\boldsymbol{s}}_{p} \otimes \widehat{\boldsymbol{v}}_{p}\right)^{T} \boldsymbol{\omega}\right)\right]+\sum_{p} \breve{b}_{p} e_{p}
\end{gathered}
$$

また，式(82)における混相のエネルギ一密度 $e$ は，式(38), (40)および式(42)を用いれば，次のように計算で きる.

$$
\begin{aligned}
e=\frac{1}{\rho} \sum_{p} \rho_{p}\left(\varepsilon_{p}+\right. & \left.\frac{1}{2} \boldsymbol{v}_{p} \cdot \boldsymbol{v}_{p}+\frac{1}{2} \boldsymbol{s}_{p} \cdot \boldsymbol{\omega}_{p}\right)=\frac{1}{\rho} \sum_{p} \rho_{p}\left[\boldsymbol{\varepsilon}_{p}+\frac{1}{2}\left(\boldsymbol{v}+\widehat{\boldsymbol{v}}_{p}\right) \cdot\left(\boldsymbol{v}+\widehat{\boldsymbol{v}}_{p}\right)+\frac{1}{2}\left(\boldsymbol{s}+\widehat{\boldsymbol{s}}_{p}\right) \cdot\left(\boldsymbol{\omega}+\widehat{\boldsymbol{\omega}}_{p}\right)\right] \\
& =\frac{1}{\rho}\left[\sum_{p} \boldsymbol{\varepsilon}_{p} \rho_{p}+\left(\frac{1}{2} \boldsymbol{v} \cdot \boldsymbol{v} \sum_{p} \rho_{p}+\boldsymbol{v} \cdot \sum_{p} \rho_{p} \widehat{\boldsymbol{v}}_{p}+\frac{1}{2} \sum_{p} \rho_{p} \widehat{\boldsymbol{v}}_{p} \cdot \widehat{\boldsymbol{v}}_{p}\right)\right. \\
& \left.+\left(\frac{1}{2} \boldsymbol{s} \cdot \boldsymbol{\omega} \sum_{p} \rho_{p}+\frac{1}{2} \boldsymbol{s} \cdot \sum_{p} \rho_{p} \widehat{\boldsymbol{\omega}}_{p}+\frac{1}{2} \sum_{p} \rho_{p} \widehat{\boldsymbol{s}}_{p} \cdot \boldsymbol{\omega}+\frac{1}{2} \sum_{p} \rho_{p} \widehat{\boldsymbol{s}}_{p} \cdot \widehat{\boldsymbol{\omega}}_{p}\right)\right] \\
=\left(\varepsilon_{\mathrm{I}}+\right. & \left.\frac{1}{2 \rho} \sum_{p} \rho_{p} \widehat{\boldsymbol{v}}_{p} \cdot \widehat{\boldsymbol{v}}_{p}+\frac{1}{2 \rho} \sum_{p} \rho_{p} \widehat{\boldsymbol{s}}_{p} \cdot \widehat{\boldsymbol{\omega}}_{p}\right)+\frac{1}{2} \boldsymbol{v} \cdot \boldsymbol{v}+\frac{1}{2} \boldsymbol{s} \cdot \boldsymbol{\omega}=\boldsymbol{\varepsilon}+\frac{1}{2} \boldsymbol{v} \cdot \boldsymbol{v}+\frac{1}{2} \boldsymbol{s} \cdot \boldsymbol{\omega}
\end{aligned}
$$

ここで，混相の内部エネルギ一密度 $\varepsilon$ および各相の単純和 $\varepsilon_{\mathrm{I}}$ をそれぞれ次式のように定義している.

$$
\varepsilon \equiv \varepsilon_{\mathrm{I}}+\frac{1}{2 \rho} \sum_{p} \rho_{p} \widehat{\boldsymbol{v}}_{p} \cdot \widehat{\boldsymbol{v}}_{p}+\frac{1}{2 \rho} \sum_{p} \rho_{p} \widehat{\boldsymbol{s}}_{p} \cdot \widehat{\boldsymbol{\omega}}_{p}, \quad \varepsilon_{\mathrm{I}} \equiv \frac{1}{\rho} \sum_{p} \rho_{p} \varepsilon_{p}
$$


ただし, 内部エネルギー密度の平均量 $\varepsilon_{\mathrm{I}}$ には式(40)を用いている(24).一方, 式(38)および式(42)を式(79)右 辺第1項に考慮すれば,

$$
\begin{gathered}
-\operatorname{div} \sum_{p}\left(\boldsymbol{q}_{p}-\boldsymbol{T}_{p}^{T} \boldsymbol{v}_{p}-\boldsymbol{M}_{p}^{T} \boldsymbol{\omega}_{p}\right)=-\operatorname{div} \sum_{p}\left[\boldsymbol{q}_{p}-\boldsymbol{T}_{p}^{T}\left(\boldsymbol{v}+\widehat{\boldsymbol{v}}_{p}\right)-\boldsymbol{M}_{p}^{T}\left(\boldsymbol{\omega}+\widehat{\boldsymbol{\omega}}_{p}\right)\right] \\
=-\operatorname{div} \sum_{p}\left[\left(\boldsymbol{q}_{p}-\boldsymbol{T}_{p}^{T} \boldsymbol{v}-\boldsymbol{M}_{p}^{T} \boldsymbol{\omega}\right)-\left(\boldsymbol{T}_{p}^{T} \widehat{\boldsymbol{v}}_{p}+\boldsymbol{M}_{p}^{T} \widehat{\boldsymbol{\omega}}_{p}\right)\right]
\end{gathered}
$$

となる．以上，式(82)および(85)を式(79)に代入すれば，次式が得られる.

$$
\begin{gathered}
\rho \dot{\boldsymbol{e}}=-\operatorname{div} \sum_{p}\left[\left(\boldsymbol{q}_{p}-\boldsymbol{T}_{p}^{T} \widehat{\boldsymbol{v}}_{p}-\boldsymbol{M}_{p}^{T} \widehat{\boldsymbol{\omega}}_{p}\right)+\rho_{p}\left(\varepsilon_{p}+\frac{1}{2} \widehat{\boldsymbol{v}}_{p} \cdot \widehat{\boldsymbol{v}}_{p}+\frac{1}{2} \widehat{\boldsymbol{s}}_{p} \cdot \widehat{\boldsymbol{\omega}}_{p}\right) \widehat{\boldsymbol{v}}_{p}\right] \\
+\operatorname{div}\left[\sum_{p}\left(\boldsymbol{T}_{p}^{T}-\rho_{p} \widehat{\boldsymbol{v}}_{p} \otimes \widehat{\boldsymbol{v}}_{p}\right)^{T} \boldsymbol{v}+\sum_{p}\left(\boldsymbol{M}_{p}^{T}-\rho_{p} \widehat{\boldsymbol{s}}_{p} \otimes \widehat{\boldsymbol{v}}_{p}\right)^{T} \boldsymbol{\omega}\right] \\
-\frac{1}{2} \operatorname{div} \sum_{p}\left[\left(\rho_{p} \widehat{\boldsymbol{\omega}}_{p} \otimes \widehat{\boldsymbol{v}}_{p}\right)^{T} \boldsymbol{s}-\left(\rho_{p} \widehat{\boldsymbol{s}}_{p} \otimes \widehat{\boldsymbol{v}}_{p}\right)^{T} \boldsymbol{\omega}\right]+\sum_{p}\left(\boldsymbol{v}_{p} \cdot \breve{\boldsymbol{p}}_{p}+\boldsymbol{\omega}_{p} \cdot \breve{\boldsymbol{l}}_{p}+\breve{\boldsymbol{\varepsilon}}_{p}-\breve{b}_{p} e_{p}\right)
\end{gathered}
$$

さらに, 式(86)右辺第3項の被微分量について, $\boldsymbol{J}_{p}=\boldsymbol{J}_{p}^{T}$ の関係を用いれば, 次式が成り立つ.

$$
\begin{aligned}
& \left(\rho_{p} \widehat{\boldsymbol{\omega}}_{p} \otimes \widehat{\boldsymbol{v}}_{p}\right)^{T} \boldsymbol{s}-\left(\rho_{p} \widehat{\boldsymbol{s}}_{p} \otimes \widehat{\boldsymbol{v}}_{p}\right)^{T} \boldsymbol{\omega}=\rho_{p}\left(\boldsymbol{s} \cdot \widehat{\boldsymbol{\omega}}_{p}-\widehat{\boldsymbol{s}}_{p} \cdot \boldsymbol{\omega}\right) \widehat{\boldsymbol{v}}_{p} \\
= & \rho_{p}\left[\left(\boldsymbol{J}_{p} \boldsymbol{\omega}\right) \cdot \widehat{\boldsymbol{\omega}}_{p}-\left(\boldsymbol{J}_{p} \widehat{\boldsymbol{\omega}}_{p}\right) \cdot \boldsymbol{\omega}\right] \widehat{\boldsymbol{v}}_{p}=\rho_{p}\left[\left(\boldsymbol{J}_{p}-\boldsymbol{J}_{p}^{T}\right) \widehat{\boldsymbol{\omega}}_{p} \cdot \boldsymbol{\omega}\right] \widehat{\boldsymbol{v}}_{p}=0
\end{aligned}
$$

式(86)右辺第4項は，式(44)より次式のように零となる.

$$
\sum_{p}\left(\boldsymbol{v}_{p} \cdot \breve{\boldsymbol{p}}_{p}+\boldsymbol{\omega}_{p} \cdot \breve{\boldsymbol{l}}_{p}+\breve{\varepsilon}_{p}-\breve{b}_{p} e_{p}\right)=0
$$

式(87)および式(88)を式(86)に代入し，式(63)および式(74)を用いれば，式(86)は次式のようになる.

$$
\rho \dot{e}=-\operatorname{div}\left(\boldsymbol{q}-\boldsymbol{T}^{T} \boldsymbol{v}-\boldsymbol{M}^{T} \boldsymbol{\omega}\right)
$$

ここでは，式(86)右辺（）内の第1項において混相の熱流束の $\boldsymbol{q}$ および熱流束の各相の単純和 $\boldsymbol{q}_{\mathrm{I}}$ をそれぞれ

$$
\boldsymbol{q} \equiv \boldsymbol{q}_{\mathrm{I}}+\sum_{p}\left[-\boldsymbol{T}_{p}^{T} \widehat{\boldsymbol{v}}_{p}-\boldsymbol{M}_{p}^{T} \widehat{\boldsymbol{\omega}}_{p}+\rho_{p} \varepsilon_{p} \widehat{\boldsymbol{v}}_{p}+\rho_{p}\left(\frac{1}{2} \widehat{\boldsymbol{v}}_{p} \cdot \widehat{\boldsymbol{v}}_{p}+\frac{1}{2} \widehat{\boldsymbol{s}}_{p} \cdot \widehat{\boldsymbol{\omega}}_{p}\right) \widehat{\boldsymbol{v}}_{p}\right], \quad \boldsymbol{q}_{\mathrm{I}} \equiv \sum_{p} \boldsymbol{q}_{p}
$$

と定義している，さらに，式(77)1および式(83)を用いれば，式(89)左辺は

$$
\rho \dot{e}=\rho\left(\varepsilon+\frac{1}{2} \boldsymbol{v} \cdot \boldsymbol{v}+\frac{1}{2} \boldsymbol{s} \cdot \boldsymbol{\omega}\right)^{\cdot}=\rho \dot{\varepsilon}+\rho \dot{\boldsymbol{v}} \cdot \boldsymbol{v}+\rho \dot{\boldsymbol{s}} \cdot \boldsymbol{\omega}
$$

のようになる．ただしここでは $\boldsymbol{J}=\boldsymbol{J}^{T}$ および $\boldsymbol{J}=\boldsymbol{I} \boldsymbol{\Omega}-\boldsymbol{\Omega} \boldsymbol{I}$ を考慮して導出した以下のような関係式が用い られている(付録参照)。ここで $\Omega$ は平均スピン角速度テンソルである.

$$
\dot{\boldsymbol{s}} \cdot \boldsymbol{\omega}=(J \boldsymbol{\omega})^{\cdot} \cdot \boldsymbol{\omega}=(\dot{J} \omega) \cdot \omega+(J \dot{\omega}) \cdot \omega=(-\Omega I \omega+J \dot{\omega}) \cdot \omega=(J \omega) \cdot \dot{\omega}=s \cdot \dot{\omega}
$$

ここで， $\boldsymbol{\Omega} \equiv \operatorname{dual} \boldsymbol{\omega}$ (または $\Omega_{i j} \equiv e_{i k j} \omega_{k}$ ) および $\boldsymbol{\omega} \times \boldsymbol{\omega}=0$ を考慮している.また，式(89)右辺（）内の第2項 および第3項は

$$
\operatorname{div}\left(\boldsymbol{T}^{T} \boldsymbol{v}\right)+\operatorname{div}\left(\boldsymbol{M}^{T} \boldsymbol{\omega}\right)=\boldsymbol{T} \cdot \operatorname{grad} \boldsymbol{v}+\boldsymbol{v} \cdot \operatorname{div} \boldsymbol{T}+\boldsymbol{M} \cdot \operatorname{grad} \boldsymbol{\omega}+\boldsymbol{\omega} \cdot \operatorname{div} \boldsymbol{M}
$$

のようになる. 式(91)および式(93)を式(89)に代入するとともに式(66)および式(76)を考慮すれば

$\rho \dot{\varepsilon}=-\operatorname{div} \boldsymbol{q}+\boldsymbol{T} \cdot \operatorname{grad} \boldsymbol{v}+\boldsymbol{M} \cdot \operatorname{grad} \boldsymbol{\omega}+(\operatorname{div} \boldsymbol{T}-\rho \dot{\boldsymbol{v}}) \cdot \boldsymbol{v}+(\operatorname{div} \boldsymbol{M}-\rho \dot{\boldsymbol{s}}) \cdot \boldsymbol{\omega}=-\operatorname{div} \boldsymbol{q}+\boldsymbol{T} \cdot \operatorname{grad} \boldsymbol{v}+\boldsymbol{M} \cdot \operatorname{grad} \boldsymbol{\omega}$ (94) が得られる. 式(94)において速度こう配 $\operatorname{grad} v$ および格子の回転角速度こう配 $\operatorname{grad} \omega$ はそれぞれ

$$
\operatorname{grad} \boldsymbol{v} \equiv \boldsymbol{L}=\boldsymbol{D}+\boldsymbol{W}, \quad \dot{\boldsymbol{\Lambda}} \equiv \operatorname{grad} \boldsymbol{\omega}
$$

のように書ける．ここで， $\boldsymbol{D}=\dot{\boldsymbol{E}}$ は変形速度(微小ひずみの速度)および $\boldsymbol{W}$ は連続体スピンであり，それぞ れ速度こう配 $\boldsymbol{L}$ の対称および反対称部分である.式(70)および式(95)を式(94)に適用し， $\boldsymbol{T} \cdot \boldsymbol{W}=0$ を考慮す れば，エネルギー方程式が

$$
\rho \dot{\varepsilon}=-\operatorname{div} \boldsymbol{q}+\boldsymbol{T} \cdot \dot{\boldsymbol{E}}+\boldsymbol{M} \cdot \dot{\boldsymbol{A}}
$$

\section{$4 \cdot 6$ エントロピー増大則}

混相に対するエントロピー増大則は，次式のように定式化できる(24).

$$
\sum_{p}\left[\frac{\bar{D}}{\bar{D} t} \int_{V} \rho_{p} \eta_{p} d v-\left(-\oint_{\mathrm{S}} \frac{\boldsymbol{q}_{p}}{\theta_{\mathrm{K}}} \cdot \boldsymbol{n} d a+\int_{\mathrm{V}} \breve{b}_{p} \eta_{p} d v\right)\right] \geq 0
$$

ここで， $\eta_{p}$ は相 $p$ のエントロピー密度， $\theta_{\mathrm{K}}$ は温度である。ただし，混相の場合も一物質点の温度 $\theta_{\mathrm{K} p}$ は 
$\theta_{\mathrm{K} p}=\theta_{\mathrm{K}}$ として一定であると考える．また，混相状態においては，相どうしの拡散によるエントロピーの 増加があるので，エントロピー増大則ははじめから和の形で表示している. 式(20)を式(97)左辺 [ ]内の第 1項に適用すれば

$$
\frac{\bar{D}}{\bar{D} t} \int_{\vee} \rho_{p} \eta_{p} d v=\int_{\vee}\left(\rho_{p} \dot{\eta}_{p}+\breve{b}_{p} \eta_{p}\right) d v
$$

のようになる。また，式(97)左辺 [ ]内の第2項に, Gaussの発散定理を適用すれば, 次式のようになる.

$$
\oint_{\mathrm{S}} \frac{\boldsymbol{q}_{p}}{\theta_{\mathrm{K}}} \cdot \boldsymbol{n} d a=\int_{\mathrm{V}} \operatorname{div} \frac{\boldsymbol{q}_{p}}{\theta_{\mathrm{K}}} d v
$$

式(98)および式(99)を式(97)に代入して，局所化すれば次式を得る.

$$
\sum_{p}\left(\rho_{p} \dot{\eta}_{p}+\operatorname{div} \frac{\boldsymbol{q}_{p}}{\theta_{\mathrm{K}}}\right) \geq 0
$$

ここで, 式(51)を式(100)左辺（）内の第1項に適用すれば次式のようになる.

$$
\sum_{p} \rho_{p} \grave{\eta}_{p}=\rho \dot{\eta}+\operatorname{div} \sum_{p} \rho_{p} \eta_{p} \widehat{\boldsymbol{v}}_{p}+\sum_{p} \breve{b}_{p} \eta_{p}
$$

ここで $\eta$ は混相状態でのエントロピー密度である. また, 式(90)を式(100)左辺（）内の第2項に代入すれば

$$
\sum_{p} \operatorname{div} \frac{\boldsymbol{q}_{p}}{\theta_{\mathrm{K}}}=\operatorname{div} \frac{1}{\theta_{\mathrm{K}}} \sum_{p} \boldsymbol{q}_{p}=\operatorname{div} \frac{1}{\theta_{\mathrm{K}}}\left\{\boldsymbol{q}+\sum_{p}\left[\boldsymbol{T}_{p}^{T} \widehat{\boldsymbol{v}}_{p}+\boldsymbol{M}_{p}^{T} \widehat{\boldsymbol{\omega}}_{p}-\rho_{p}\left(\varepsilon_{p}+\frac{1}{2} \widehat{\boldsymbol{v}}_{p} \cdot \widehat{\boldsymbol{v}}_{p}+\frac{1}{2} \widehat{\boldsymbol{s}}_{p} \cdot \widehat{\boldsymbol{\omega}}_{p}\right) \widehat{\boldsymbol{v}}_{p}\right]\right\}
$$

のようになる.さらに，式(44)より次式が成り立つ.

$$
\sum_{p} \breve{b}_{p} \eta_{p}=0
$$

式(101)，(103)および式(102)を式(100)に代入すれば，次式が導出される.

$$
\rho \dot{\eta}+\operatorname{div} \frac{\boldsymbol{q}}{\theta_{\mathrm{K}}}+\operatorname{div} \frac{1}{\theta_{\mathrm{K}}} \sum_{p}\left[\boldsymbol{T}_{p}^{T} \widehat{\boldsymbol{v}}_{p}+\boldsymbol{M}_{p}^{T} \widehat{\boldsymbol{\omega}}_{p}-\rho_{p}\left(\varepsilon_{p}-\theta_{\mathrm{K}} \eta_{p}+\frac{1}{2} \widehat{\boldsymbol{v}}_{p} \cdot \widehat{\boldsymbol{v}}_{p}+\frac{1}{2} \widehat{\boldsymbol{s}}_{p} \cdot \widehat{\boldsymbol{\omega}}_{p}\right) \widehat{\boldsymbol{v}}_{p}\right] \geq 0
$$

ここで，自由エネルギー密度 $\psi_{p}$ を $\psi_{p} \equiv \varepsilon_{p}-\theta_{\mathrm{K}} \eta_{p}$ と定義すれば，式(104)左辺の $[$ ]内は，

$$
\begin{gathered}
\boldsymbol{T}_{p}^{T} \widehat{\boldsymbol{v}}_{p}+\boldsymbol{M}_{p}^{T} \widehat{\boldsymbol{\omega}}_{p}-\rho_{p}\left(\varepsilon_{p}-\theta_{\mathrm{K}} \eta_{p}+\frac{1}{2} \widehat{\boldsymbol{v}}_{p} \cdot \widehat{\boldsymbol{v}}_{p}+\frac{1}{2} \widehat{\boldsymbol{s}}_{p} \cdot \widehat{\boldsymbol{\omega}}_{p}\right) \widehat{\boldsymbol{v}}_{p} \\
=-\rho_{p} \psi_{p} \widehat{\boldsymbol{v}}_{p}-\frac{1}{2} \rho_{p}\left(\widehat{\boldsymbol{v}}_{p} \cdot \widehat{\boldsymbol{v}}_{p}\right) \widehat{\boldsymbol{v}}_{p}-\frac{1}{2} \rho_{p}\left(\widehat{\boldsymbol{s}}_{p} \cdot \widehat{\boldsymbol{\omega}}_{p}\right) \widehat{\boldsymbol{v}}_{p}+\boldsymbol{T}_{p}^{T} \widehat{\boldsymbol{v}}_{p}+\boldsymbol{M}_{p}^{T} \widehat{\boldsymbol{\omega}}_{p}
\end{gathered}
$$

のように表せる. さらに, 応力 $\boldsymbol{T}_{p}$ を静水圧応力 $\sigma_{m p}$ および偏差応力 $\boldsymbol{T}_{p}^{\prime}$ を用いて $\boldsymbol{T}_{p} \equiv \sigma_{m p} \boldsymbol{1}+\boldsymbol{T}_{p}^{\prime T}$ のように分 解すれば，式(105)の右辺は，

$$
\begin{gathered}
-\rho_{p} \psi_{p} \widehat{\boldsymbol{v}}_{p}-\frac{1}{2} \rho_{p}\left(\widehat{\boldsymbol{v}}_{p} \cdot \widehat{\boldsymbol{v}}_{p}\right) \widehat{\boldsymbol{v}}_{p}-\frac{1}{2} \rho_{p}\left(\widehat{\boldsymbol{s}}_{p} \cdot \widehat{\boldsymbol{\omega}}_{p}\right) \widehat{\boldsymbol{v}}_{p}+\boldsymbol{T}_{p}^{T} \widehat{\boldsymbol{v}}_{p}+\boldsymbol{M}_{p}^{T} \widehat{\boldsymbol{\omega}}_{p} \\
=-\rho_{p}\left[\left(\psi_{p}-\frac{\sigma_{m p}}{\rho_{p}}\right)+\frac{1}{2}\left(\widehat{\boldsymbol{v}}_{p} \cdot \widehat{\boldsymbol{v}}_{p}\right)+\frac{1}{2}\left(\widehat{\boldsymbol{s}}_{p} \cdot \widehat{\boldsymbol{\omega}}_{p}\right)\right] \widehat{\boldsymbol{v}}_{p}+\boldsymbol{T}_{p}^{\prime T} \widehat{\boldsymbol{v}}_{p}+\boldsymbol{M}_{p}^{T} \widehat{\boldsymbol{\omega}}_{p}=-\rho_{p} \mu_{p} \widehat{\boldsymbol{v}}_{p}+\boldsymbol{T}_{p}^{\prime T} \widehat{\boldsymbol{v}}_{p}+\boldsymbol{M}_{p}^{T} \widehat{\boldsymbol{\omega}}_{p}
\end{gathered}
$$

のように書ける．ここで,

$$
\mu_{p} \equiv \mu_{p \mathrm{I}}+\frac{1}{2} \widehat{\boldsymbol{v}}_{p} \cdot \widehat{\boldsymbol{v}}_{p}+\frac{1}{2} \widehat{\boldsymbol{s}}_{p} \cdot \widehat{\boldsymbol{\omega}}_{p}, \quad \mu_{p \mathrm{I}} \equiv \psi_{p}-\sigma_{m p} / \rho_{p}
$$

であり， $\mu_{p 1}$ は相 $p$ の化学ポテンシャル (Gibbs関数密度)である. 式(105)および式(106)を式(104)に代入し， 式(57)を用いて書き改めれば，次式が得られる.

$$
\rho \dot{\eta}+\operatorname{div} \frac{\boldsymbol{q}}{\theta_{\mathrm{K}}}-\operatorname{div} \sum_{p} \frac{\mu_{p} \hat{\boldsymbol{j}}_{p}}{\theta_{\mathrm{K}}}+\operatorname{div} \sum_{p} \frac{\boldsymbol{k}_{p}}{\theta_{\mathrm{K}}} \geq 0
$$

ここで, 式(108)の左辺第3項の被微分量は相転移の局所エントロピー流束であり, 一方， $\boldsymbol{k}_{p}$ は

$$
\boldsymbol{k}_{p} \equiv \boldsymbol{T}_{p}^{\prime^{T}} \widehat{\boldsymbol{v}}_{p}+\boldsymbol{M}_{p}^{T} \widehat{\boldsymbol{\omega}}_{p}
$$

のように定義される混相の力学的なエントロピー流束である. 混相状態の局所エントロピー流束を

$$
\hat{\boldsymbol{s}} \equiv \frac{\boldsymbol{q}}{\theta_{\mathrm{K}}}-\sum_{p} \frac{\mu_{p} \widehat{\hat{\boldsymbol{j}}}_{p}}{\theta_{\mathrm{K}}}+\sum_{p} \frac{\boldsymbol{k}_{p}}{\theta_{\mathrm{K}}}
$$

と定義すれば，エントロピー増大則は 


$$
\rho \dot{\eta}+\operatorname{div} \hat{\boldsymbol{s}} \geq 0
$$

のように書き改められる。本節で定式化した式(110)および式(111)のエントロピー増大則を用いれば，熱力 学的議論を通して流束の構成式を導出することができる。また, Phase-fieldモデルを構築するためには, 秩 序変数および結晶方位で表記された質量保存則およびスピン角運動量保存則に，それぞれ秩序流束および 方位流束の構成式を代入して基礎方程式系を得る必要がある。ただし，このような流束の構成式の導出な らびにPhase-fieldモデルの構築については次報に委ねる.

\section{5. 結}

前報で定式化した相 $p$ に対する離散的保存則に対し，代表体積要素を連続体の一物質点に帰着させること で，相 $p$ に対する連続体としての質量，運動量，角運動量およびエネルギーの保存則を導出した。また，得 られた保存則を通常の手順で局所化するとともに，局所形の保存則を相にわたって和を取り，平均化を施 して混相の保存則を得た。さらに，得られた諸保存則に基づいてエントロピー増大則を定式化した。以上 の結果，以下の結論を得た.

(1) 混相に対する質量および運動量の保存則は, いずれも単純体に対するものと形式上一致する. 一方, 再結晶相 (単相)の質量保存則において，密度を体積分率で表示し直せば，秩序変数に対する釣合い方 程式が得られる.

（2）バルクの角運動量保存則からは単純体の角運動量方程式が得られ，混相のCauchy応力は対称性を保つ. 一方, 格子の角運動量方程式からは結晶方位に対するPhase-field方程式に相当する発展式が導出される.

(3) 混相のエネルギー保存則およびエントロピー増大則を定式化すれば，混相における相転移のエントロ ピー流束は化学ポテンシャルと相対質量流束を用いて定義される.

\section{謝辞}

本研究は, 平成23年度科学研究費 (基盤研究C) の補助を得て実施したものであることを記し, 謝意を表 す.

\section{付 録}

\section{慣性モーメントテンソル}

RVE内で平均化された慣性モーメントの物質時間微分 $\boldsymbol{j}$ を求めるために，まず $\boldsymbol{J}^{(k)}$ を定義する。格子要 素を棒要素(図6)としてモデル化し, その重心位置を固定して考えれば, 格子要素のスピン角運動量は, 次 式で表わされる.

$$
\int_{V_{1}} \boldsymbol{\xi}^{\prime(k)} \times \rho^{\prime} \boldsymbol{v}^{\prime(k)} d v_{l}=\int_{V_{1}} \boldsymbol{\xi}^{\prime(k)} \times \rho^{\prime} \boldsymbol{\Omega}^{(k)} \boldsymbol{\xi}^{\prime(k)} d v_{l}
$$

ここで， $\boldsymbol{\xi}^{\prime(k)}$ は $k$ 番目の格子要素の重心から要素内の任意点までの位置ベクトル， $\rho^{\prime}$ は格子要素の質量密 度および $\boldsymbol{\Omega}^{(k)}$ は重心まわりのスピン角速度テンソルであり， $\boldsymbol{\Omega}^{(k)} \equiv \operatorname{dual} \boldsymbol{\omega}^{(k)}$ が成り立つ ${ }^{(25)}$. また， $v_{l}$ は格 子要素1つの体積である。ここで， $\boldsymbol{I}^{(k)} \equiv \boldsymbol{\xi}^{(k)} \otimes \boldsymbol{\xi}^{(k)}$ と定義すれば，式(112)右辺の被積分関数は

$$
\begin{aligned}
\left(\xi^{\prime(k)} \times \rho^{\prime} \boldsymbol{\Omega}^{(k)} \xi^{\prime(k)}\right)_{i}=\rho^{\prime} e_{i j k} \xi_{j}^{\prime(k)} \Omega_{k l}^{(k)} \xi_{l}^{\prime(k)} & =\rho^{\prime} e_{i j k} e_{k m l} \xi_{j}^{\prime(k)} \xi_{l}^{\prime(k)} \omega_{m}^{(k)}=\rho^{\prime}\left(\delta_{i m}^{\prime(k)} \delta_{j l}^{\prime(k)}-\delta_{i l}^{\prime(k)} \delta_{j m}^{\prime(k)}\right) \xi_{j}^{\prime(k)} \xi_{l}^{\prime(k)} \omega_{m}^{(k)} \\
= & \rho^{\prime}\left[\left(\xi_{j}^{\prime(k)} \xi_{j}^{\prime(k)}\right) \delta_{i m}^{\prime(k)}-\xi_{i}^{\prime(k)} \xi_{m}^{\prime(k)}\right] \omega_{m}^{(k)}=\rho^{\prime}\left[\left(\xi_{k}^{\prime(k)} \xi_{k}^{\prime(k)}\right) \delta_{i j}^{\prime(k)}-\xi_{i}^{\prime(k)} \xi_{j}^{\prime(k)}\right] \omega_{j}^{(k)}=\left(\rho^{\prime} \boldsymbol{J}^{\prime(k)} \boldsymbol{\omega}^{(k)}\right)_{i}
\end{aligned}
$$

となる。ここで， $\boldsymbol{J}^{(k)}$ は次式のとおりである.

$$
\boldsymbol{J}^{\prime(k)} \equiv\left(\operatorname{tr} \boldsymbol{I}^{(k)}\right) \boldsymbol{I}-\boldsymbol{I}^{(k)}
$$

式(113)を式(112)に代入し, 領域 $v_{l}$ 内で $\omega^{(k)}$ が一定であることを考慮すれば

$$
\int_{V_{1}} \boldsymbol{\xi}^{\prime(k)} \times \rho^{\prime} \boldsymbol{v}^{\prime(k)} d v_{l}=\int_{V_{1}} \rho^{\prime(k)} \boldsymbol{J}^{\prime(k)} \boldsymbol{\omega}^{(k)} d v_{l}=m^{(k)} \boldsymbol{J}^{(k)} \boldsymbol{\omega}^{(k)}
$$

のようになる.ここで $\boldsymbol{J}^{(k)}$ は

$$
\boldsymbol{J}^{(k)} \equiv \frac{1}{m^{(k)}} \int_{V_{1}} \rho^{\prime(k)} \boldsymbol{J}^{(k)} d v_{l}
$$




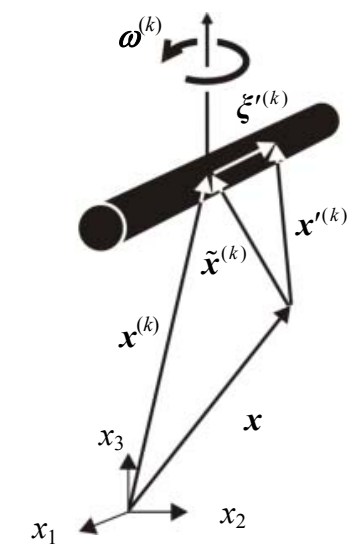

Fig. 6 Illustration of lattice element

である，一方，式(114)を考慮して， $\boldsymbol{J}^{(k)}$ を $\boldsymbol{I}^{(k)}$ で書けば

$$
\left.\boldsymbol{J}^{(k)} \equiv\left\langle\boldsymbol{J}^{\prime(k)}\right\rangle_{\mathrm{m}_{1}}=<\left(\operatorname{tr} \boldsymbol{I}^{(k)}\right) \boldsymbol{I}-\boldsymbol{I}^{\prime(k)}\right\rangle_{\mathrm{m}_{1}}=\left(\operatorname{tr}\left\langle\boldsymbol{I}^{\prime(k)}\right\rangle_{\mathrm{m}_{\mathrm{l}}}\right) \boldsymbol{I}-\left\langle\boldsymbol{I}^{(k)}\right\rangle_{\mathrm{m}_{1}}=\left(\operatorname{tr} \boldsymbol{I}^{(k)}\right) \boldsymbol{I}-\boldsymbol{I}^{(k)}
$$

のようになる，ここで， < $>_{\mathrm{m}_{1}}$ は格子要素内での質量平均量を表わす．したがって，式(117)の両辺を物質 時間微分すれば次式が得られる.

$$
\dot{\boldsymbol{J}}^{(k)}=\left(\operatorname{tr} \dot{\boldsymbol{I}}^{(k)}\right) \boldsymbol{1}-\dot{\boldsymbol{I}}^{(k)}
$$

ここで， $\boldsymbol{I}^{(k)}$ の物質時間微分 $\dot{\boldsymbol{I}}^{(k)}$ は

$$
\dot{\boldsymbol{I}}^{(k)}=\left(\left\langle\boldsymbol{I}^{\prime(k)}\right\rangle_{\mathrm{m}_{1}}\right)^{\cdot}=\left(\left\langle\boldsymbol{\xi}^{\prime(k)} \otimes \boldsymbol{\xi}^{\prime(k)}\right\rangle_{\mathrm{m}_{1}}\right)^{\cdot}=\left\langle\dot{\boldsymbol{\xi}}^{\prime(k)} \otimes \boldsymbol{\xi}^{\prime(k)}\right\rangle_{\mathrm{m}_{1}}+\left\langle\boldsymbol{\xi}^{\prime(k)} \otimes \dot{\boldsymbol{\xi}}^{\prime(k)}\right\rangle_{\mathrm{m}_{1}}
$$

のようになる，ただし， $\boldsymbol{\xi}^{(k)}$ は格子のスピン運動に対する直交テンソル $\boldsymbol{R}^{(k)}$ および初期の位置ベクトル $\boldsymbol{\xi}_{0}^{\prime(k)}$ を用いて次式のように書ける ${ }^{(25)}$.

$$
\boldsymbol{\xi}^{\prime(k)}=\boldsymbol{R}^{(k)} \boldsymbol{\xi}_{0}^{\prime(k)}
$$

したがって，式(120)の両辺を物質時間微分すれば,

$$
\dot{\boldsymbol{\xi}}^{\prime(k)}=\dot{\boldsymbol{R}}^{(k)} \boldsymbol{\xi}_{0}^{\prime(k)}=\dot{\boldsymbol{R}}^{(k)} \boldsymbol{R}^{(k) T} \boldsymbol{\xi}^{(k)}=\boldsymbol{\Omega}^{(k)} \boldsymbol{\xi}^{(k)}
$$

なる関係式が得られる。ここで， $\boldsymbol{\Omega}^{(k)} \equiv \dot{\boldsymbol{R}}^{(k)} \boldsymbol{R}^{(k) T}$ と定義している

$$
\begin{aligned}
\dot{\boldsymbol{I}}^{(k)}=\left\langle\left(\boldsymbol{\Omega}^{(k)} \boldsymbol{\xi}^{\prime(k)}\right) \otimes \boldsymbol{\xi}^{\prime(k)}>_{\mathrm{m}_{1}}+\right. & <\boldsymbol{\xi}^{\prime(k)} \otimes\left(\boldsymbol{\Omega}^{(k)} \boldsymbol{\xi}^{\prime(k)}\right)>_{\mathrm{m}_{1}}=\boldsymbol{\Omega}^{(k)}<\boldsymbol{\xi}^{\prime(k)} \otimes \boldsymbol{\xi}^{\prime(k)}>_{\mathrm{m}_{1}}+\left\langle\boldsymbol{\xi}^{\prime(k)} \otimes \boldsymbol{\xi}^{\prime(k)}>_{\mathrm{m}_{1}} \boldsymbol{\Omega}^{(k) T}\right. \\
& =\boldsymbol{\Omega}^{(k)} \boldsymbol{I}^{(k)}+\boldsymbol{I}^{(k)} \boldsymbol{\Omega}^{(k) T}=\boldsymbol{\Omega}^{(k)} \boldsymbol{I}^{(k)}-\boldsymbol{I}^{(k)} \boldsymbol{\Omega}^{(k)}
\end{aligned}
$$

のようになる．式(122)の両辺の対角成分の和をとれば， $\operatorname{tr} \dot{\boldsymbol{I}}^{(k)}$ が以下のように計算される.

$$
\operatorname{tr} \dot{\boldsymbol{I}}^{(k)}=\operatorname{tr}\left(\boldsymbol{\Omega}^{(k)} \boldsymbol{I}^{(k)}\right)-\operatorname{tr}\left(\boldsymbol{I}^{(k)} \boldsymbol{\Omega}^{(k)}\right)=0
$$

式(122)および式(123)を式(118)に代入すれば

$$
\dot{\boldsymbol{J}}^{(k)}=-\dot{\boldsymbol{I}}^{(k)}=\boldsymbol{I}^{(k)} \boldsymbol{\Omega}^{(k)}-\boldsymbol{\Omega}^{(k)} \boldsymbol{I}^{(k)}
$$

となる.さらに，式(124)で $\boldsymbol{\Omega}^{(k)}=\boldsymbol{\Omega}+\tilde{\boldsymbol{\Omega}}^{(k)}$ と表し，RVE内で $\boldsymbol{J}^{(k)}$ を平均化すれば,

$$
\dot{\boldsymbol{J}}=\left\langle\dot{\boldsymbol{J}}^{(k)}\right\rangle_{\mathrm{m}}=\left\langle\boldsymbol{I}^{(k)} \boldsymbol{\Omega}^{(k)}-\boldsymbol{\Omega}^{(k)} \boldsymbol{I}^{(k)}\right\rangle_{\mathrm{m}}=\left\langle\boldsymbol{I}^{(k)}\right\rangle_{\mathrm{m}} \boldsymbol{\Omega}-\boldsymbol{\Omega}\left\langle\boldsymbol{I}^{(k)}\right\rangle_{\mathrm{m}}+\left\langle\boldsymbol{I}^{(k)} \tilde{\boldsymbol{\Omega}}^{(k)}\right\rangle_{\mathrm{m}}-\left\langle\tilde{\boldsymbol{\Omega}}^{(k)} \boldsymbol{I}^{(k)}\right\rangle_{\mathrm{m}}
$$

となる。ここで， $\boldsymbol{\Omega}$ およびは $\tilde{\boldsymbol{\Omega}}^{(k)}$ はそれぞれ $\boldsymbol{\Omega}^{(k)}$ の質量平均およびゆらぎ(変動量)であり， $<>_{\mathrm{m}}$ は RVE内での質量平均量を表わす. 次に, $\boldsymbol{I}^{(k)}$ を $\boldsymbol{I}^{(k)}=<\boldsymbol{I}^{(k)}>_{m}+\tilde{\boldsymbol{I}}^{(k)} \equiv \boldsymbol{I}+\tilde{\boldsymbol{I}}^{(k)}$ のようにRVE内の平均量とゆら ぎに分解すれば，式(125)は次のようになる.

$$
\dot{\boldsymbol{J}}=(\boldsymbol{I} \boldsymbol{\Omega}-\boldsymbol{\Omega} \boldsymbol{I})+<\tilde{\boldsymbol{I}}^{(k)} \tilde{\boldsymbol{\Omega}}^{(k)}-\tilde{\boldsymbol{\Omega}}^{(k)} \tilde{\boldsymbol{I}}^{(k)}>_{\mathrm{m}}
$$

通常，ゆらぎの相関は必ずしも小さいとは限らないが，I は格子要素の長さの2乗オーダーであるのに対し， $\tilde{\boldsymbol{I}}^{(k)}$ は平衡位置での原子振動の振幅の2乗オーダーであるから, 右辺の< > $>$ は右辺の $(\quad)$ に対して高次の微 小量として無視できる。したがって，式(126)は次式のように書き改められる.

$$
\dot{J}=\boldsymbol{I} \boldsymbol{\Omega}-\boldsymbol{\Omega I}
$$

さらに， $\boldsymbol{\omega}$ を式(127)に乗じ， $(\boldsymbol{\Omega} \boldsymbol{\omega})_{i}=\Omega_{i j} \omega_{j}=e_{i k j} \omega_{k} \omega_{j}=0$ なる関係を用いれば，次式を得る.

$$
\dot{J} \omega=I \Omega \omega-\Omega I \omega=-\Omega I \omega
$$

\section{文献}

(1) Humphreys, F.J. and Hatherly, M., "Recrystallization and Related Annealing Phenomena”, (2004), Elsevier. 
(2) Humphreys, F.J. and Chan, H.M., "Discontinuous and Continuous Annealing Phenomena in Aluminum-nickel Alloy” , Material Science and Technology, Vol. 12, (1996), pp. 143-148.

(3) Bailey, J.E. and Hirsch, P.B., "The Recrystallization Process in Some Polycrystalline Metals", Proceedings of The Royal Society of London Series A, Vol. 267, No. 1328, (1962), pp. 11-30.

(4) Kobayashi, R., "Modeling and Numerical Simulations of Dendritic Crystal Growth", Physica D, Vol. 63, (1993), pp.410-423.

(5) Miyazaki, T., Koyama, T., Kozakai, T., "Computer Simulations of The Phase Transformation in Real Alloy Systems Based on The Phase Field Method”, Materials Science and Engineering, A312 (2001), pp. 38-49.

(6) 上原拓也, 福井基支, 大野信忠, “フェーズフィールドモデルによる凝固組織形成と応力分布の数值解析”, 材料, Vol. 57, No. 3, (2008), pp. 231-236.

(7) Provatas, N. and Elder, K., "Phase-Field Methods in Materials Science and Engineering”, (2010), pp. 68-69, WILEYVCH Verlag GmbH \& Co. KGaA.

(8) Warren, J.A., Carter, W.C. and Kobayashi, R., “A Phase Field Model of Impingement of Solidifying Particles”, Physica $A$, Vol. 261, (1998), pp. 159-166.

(9) Steinbach, I., Pezzolla, F., Nestler, B., Seeßelberg, M., Prieler, R., Schmitz, G.J. and Rezende, J.L.L., “A Phase Field Concept for Multiphase System”, Physica D, Vol. 94, (1996), pp. 135-147.

(10) Kim, S.G., Kim, D.I, Kim, W.T. and Park, Y.B., "Computer Simulations of Two-dimensional and Three-dimensional Ideal Grain Growth”, Physical Review E, Vol. 74, (2006), p. 061605.

(11) Takaki, T., Yamanaka, A., Higa, Y. and Tomita, Y., "Phase-Field Model During Static Recrystallization Based on Crystal-Plasticity Theory", Journal of Computer-Aided Materials Design, Vol. 14, (2007), pp. 75-84.

(12) Wang, Y. and Li, J., "Phase Field Modeling of Defects and Deformation", Acta Metarialia, Vol. 58, (2010), pp. 12121235.

(13) 井上達雄・田中喜久昭・長岐滋, ”固体力学と相変態の解析”, (1975), 大河出版.

(14) Wang, S.-L., Sekerka, R.F., Wheeler, A.A., Murray, B.T., Coriell, S.R., Braun, R.J. and McFadden, G.B., “Thermodynamically-consistent Phase-field Models for Solidification”, Physica D, Vol. 69, (1993), pp. 189-200.

(15) Kröner, E., "Mechanics of Generalized Continua", (1968), all, Springer.

(16) Toupin, R.A., "Elastic Materials with Couple-Stresses", Archive for Rational Mechanics and Analysis, Vol. 11, (1962), pp. 385-414.

(17) Noll, W., "On The Foundation of The Mechanics of Continuous Media”, Carnegie Institute of Technology, Department of Mathematics report, No. 17, (1957), pp. 28-31.

(18) Eringen, A.C. and Suhubi, E.S., "Nonlinear Theory of Simple Micro-Elastic Solids-I", International Journal of Engineering Science, Vol. 2, (1964), pp. 189-203.

(19) Eringen, A.C. and Suhubi, E.S., "Nonlinear Theory of Simple Micro-Elastic Solids-II”, International Journal of Engineering Science, Vol. 2, (1964), pp. 389-404.

(20) Eringen, A.C., "Linear Theory of Micropolar Elasticity”, Journal of Mathematics and Mechanics, Vol. 15, No. 6, (1966), pp. 909-923.

(21) Kafader, C.B. and Eringen, A.C., "Micropolar Media - I The Classical Theory", International Journal of Engineering Science, Vol. 9, (1971), pp. 271-305.

(22) Ericksen, J.L. and Truesdell, C., "Exact Theory of Stress and Strain in Rod and Shells", Archive for Rational Mechanics and Analysis, Vol. 1, (1958), pp. 295-323.

(23) Green, A.E. and Rivlin, R.S., "Multipolar Continuum Mechanics", Archive for Rational Mechanics and Analysis, Vol. 17, (1964), pp. 113-147.

(24) Eringen, A. C., “Continuum Physics”, Vol. III, (1976), pp.2-127, Academic Press.

(25) 村松眞由, 青柳吉輝, 志澤一之, “再結晶現象に対する結晶格子スケールの離散的保存則の定式化”, 日本機械学会 論文集 A 編, Vol. 77, No. 780 (2011), pp. 1304-1319.

(26) Callister, W.D., “Materials Science and Engineering”, (2007), pp. 312-313, John Wiley and Sons, Inc..

(27) 古林英一, “再結晶と材料組織”, (2000), pp. 3-10, 内田老鶴圃. 